\title{
Liefern Jugendliche valide Informationen zum Bildungsstand ihrer Eltern in standardisierten Erhebungen? Befunde zu Schülerinnen und Schülern der 9. Jahrgangsstufe in Deutschland
}

\author{
Till Hovestadt (D) Thorsten Schneider
}

Eingegangen: 30. Januar 2020 / Überarbeitet: 26. Oktober 2020 / Angenommen: 25. März 2021 / Online publiziert: 3. Mai 2021

(C) Der/die Autor(en) 2021

Zusammenfassung Die Qualität der Angaben, die Jugendliche zu den Bildungsabschlüssen ihrer Eltern in einer standardisierten Befragung liefern, wird unter Heranziehung der kognitiven Theorie des Antwortverhaltens untersucht. Hierzu werden Daten des Nationalen Bildungspanels aus der 9. Jahrgangsstufe des Schuljahres 2009/10 mit multinomialen Logit-Modellen analysiert. Ungefähr jeder fünfte bzw. vierte Jugendliche liefert keine Angabe zum Abschluss der Mutter bzw. des Vaters, jeder vierte abweichende und nur jeder zweite übereinstimmende Angaben. Theoriekonform geht ein größerer Wortschatz mit weniger Fehl- und mehr konsistenten Angaben einher. Von Eltern im Ausland erworbene Abschlüsse sind entweder seltener kognitiv verankert oder können den vorgegebenen Antwortkategorien nicht zugeordnet werden. Entgegen theoretischer Annahmen und bisheriger Forschung sind Gewissenhaftigkeit und Diskussionen mit Eltern für die Qualität der Antworten unbedeutend. Vergleichende Auswertungen zu sozialen Ungleichheiten in Mathematikkompetenzen zeigen, dass diese statistisch und inhaltlich signifikant niedriger ausfallen, wenn anstelle der Selbstberichte von Eltern Proxy-Angaben der Jugendlichen verwendet werden.

Schlüsselwörter Schülerbefragung · Proxy-Angaben · Antwortverhalten · Messfehler · Bildungsungleichheit

Der vorliegende Artikel stellt eine inhaltliche und methodisch-statistische Weiterentwicklung der Bachelorarbeit „Liefern Schülerinnen und Schüler akkurate Informationen zum sozioökonomischen Status ihrer Eltern? Bachelorarbeit zur Qualität von Proxy-Angaben“ (2019) des Erstautors Till Hovestadt dar. Die Bachelorarbeit wurde von Prof. Dr. Thorsten Schneider betreut und im Jahr 2019 am Institut für Soziologie der Universität Leipzig vorgelegt.

T. Hovestadt $(\bowtie) \cdot$ Prof. Dr. T. Schneider Institut für Soziologie, Universität Leipzig, Beethovenstr. 15, 04107 Leipzig, Deutschland E-Mail: th92suro@studserv.uni-leipzig.de

Prof. Dr. T. Schneider

E-Mail: thorsten.schneider@uni-leipzig.de 


\title{
Can adolescents provide valid information on parents' educational attainment in standardized surveys? Results from a survey conducted in grade 9 in Germany
}

\begin{abstract}
The paper examines the accuracy of students' reports on parental education and discusses the cognitive model of survey response. For the empirical part, we used data from the German National Educational Panel Study collected in grade 9 in 2009/10, and analyzed students' and parents' reports using multinomial logit models. About 20 to $25 \%$ of students did not provide any information on their mother's or father's educational attainment; about $25 \%$ provided answers that were inconsistent with parents' reports; and close to 50\% answered consistently with their parents. In line with theory, better vocabulary skills were connected with lower item nonresponse and a higher number of correct answers. Many students did not answer if their parents had completed their education abroad: Either students did not know the answer or were not able to give the German equivalent. Contrary to theoretical arguments and previous findings, the results suggest that conscientiousness and frequent discussions with parents are irrelevant. Additionally, we compared the degree of social inequality in numeracy using students' and parents' reports on parental education. The results show that models using students' reports significantly underestimate educational inequalities.
\end{abstract}

Keywords Educational inequality · Measurement error · Proxy report · Student survey $\cdot$ Survey response

\section{Einleitung}

Verschiedene Bereiche der empirischen Bildungsforschung haben Ungleichheiten nach der sozialen Herkunft als zentrales Untersuchungsthema. Dies setzt voraus, dass Indikatoren zur sozialen Herkunft - wie alle Informationen - valide erhoben werden. Werden Elternbefragungen eingesetzt, nehmen viele Eltern daran nicht teil (Engzell und Jonsson 2015; Lien et al. 2001; West et al. 2001), was zu unvollständigen Daten führt. Da in Deutschland die Rücklaufquote der Elternbefragung in der Internationalen Grundschulleseuntersuchung (IGLU/PIRLS) kontinuierlich von anfänglich 89\% im Jahr 2001 auf 72\% im Jahr 2016 sank (Hußmann et al. 2017, S. 62), vermuten wir, dass es zunehmend schwieriger werden könnte, Eltern zur Teilnahme zu bewegen. Des Weiteren gehen mit zusätzlichen Elternbefragungen höhere zeitliche und finanzielle Kosten einher. Nonresponse und höhere Kosten sind mögliche Gründe, auf Elternbefragungen zu verzichten und stattdessen bei Schul-, aber auch sonstigen Erhebungen Jugendliche als alternative Auskunftsquelle zur sozialen Lage der Eltern heranzuziehen. Sie liefern dann stellvertretend Informationen, sog. ProxyAngaben. So setzten im Programme for International Student Assessment (PISA) 2015 nur 18 Länder, darunter auch Deutschland, einen Elternfragebogen ein. In der Trends in International Mathematics and Science Study (TIMSS) wurden in der 8. Jahrgangsstufe in keinem einzigen Land Eltern befragt (Hooper et al. 2017, S. 63; OECD 2017, S. 331f.). Da Proxy-Angaben aus zweiter Hand stammen, stellt sich 
die Frage nach ihrer Validität. Einige Studien ziehen diese im Fall von Schülerinnen und Schülern (SuS) als Auskunftspersonen in Zweifel (Engzell und Jonsson 2015; Jerrim und Micklewright 2014). Da invalide Angaben die Ergebnisse von Analysen zu sozialer Ungleichheit verzerren könnten, sind verlässliche Forschungsergebnisse zur Qualität der Angaben durch SuS von großer Bedeutung (vgl. Kreuter et al. 2006, S. $3471 \mathrm{f}$.).

Wichtige Beiträge zur Qualität von SuS-Angaben haben u.a. Kreuter et al. geliefert (Kreuter et al. 2006, 2010; Maaz et al. 2006). Sie haben auf Basis einer deutschlandspezifischen Ergänzungsstichprobe von PISA 2000 das Ausmaß der Eltern-Kind-Übereinstimmung verschiedener Angaben zur sozialen Herkunft (schulischer und beruflicher Bildungsabschluss sowie Berufsgruppe von Mutter und Vater) aufgezeigt, unter Rückgriff auf die kognitive Theorie des Antwortverhaltens (Tourangeau et al. 2000) Analysen zu möglichen Einflussfaktoren auf übereinstimmende Angaben durchgeführt und Ergebnisse zur Unterschätzung von sozialen Ungleichheiten in Mathematikkenntnissen bei Verwendung von SuS-Angaben im Vergleich zu Elternangaben berichtet.

Der folgende Beitrag schließt hieran an und liefert zunächst einen Überblick zum bisherigen Forschungsstand. Anschließend stellen wir die kognitive Theorie des Antwortverhaltens vor, wie sie von Tourangeau et al. (2000) vorgeschlagen und in verschiedenen Studien zur Qualität von SuS-Angaben rezipiert wurde (Kreuter et al. 2010; Maaz et al. 2006; Nusser et al. 2016). Im Vergleich zu bisherigen Studien verwenden wir zum einen aktuellere Daten und wir gehen weiteren Möglichkeiten zur Prüfung der kognitiven Theorie des Antwortverhaltens nach, um ein umfassenderes Bild zu den Antwortprozessen in Umfragen als auch zu systematischen Verzerrungen zu erhalten. Die Hypothesen werden anhand von Daten, die im Schuljahr 2010/11 in der 9. Jahrgangsstufe erhoben wurden, überprüft. Wir untersuchen mit Hilfe multinomialer logistischer Regressionen potenzielle Einflussfaktoren auf das Ausbleiben von Antworten und das Liefern von Angaben, die von denen der Eltern abweichen. Um abzuschätzen, wie stark der Effekt der sozialen Herkunft auf die Mathematikkenntnisse davon abhängt, von wem die Informationen zu den Bildungsabschlüssen der Eltern stammen, werden die Regressionskoeffizienten aus linearen Regressionen auf signifikante Unterschiede getestet. Der Beitrag endet mit einer Zusammenfassung der zentralen Befunde und Schlussfolgerungen für zukünftige Erhebungen.

\section{Forschungsstand, Theorie, Hypothesen und Forschungsfrage}

\subsection{Bisherige Befunde zum Ausmaß der Übereinstimmung von Eltern und SuS-Angaben zur sozialen Herkunft}

Der Forschungsstand zur Qualität der Angaben, die SuS zur sozialen Herkunft liefern, basiert auf Studien mit unterschiedlichen Erhebungs- und Auswertungsmethoden zu verschiedenen Ländern. Diese Studien gelangen z. T. zu widersprüchlichen Schlussfolgerungen, beziehen sich aber auch auf unterschiedliche Indikatoren der sozialen Herkunft wie schulische und berufliche Bildungsabschlüsse, Beruf oder An- 
zahl der Bücher im Haushalt. Um ein breites Bild zu erhalten, stellen wir Analysen zu verschiedenen Indikatoren der sozialen Herkunft vor. In nahezu allen berichteten Studien wird die Qualität der Angaben der SuS durch einen Abgleich mit den Angaben ihrer Eltern oder eines Elternteils bestimmt. Mangels ,wahrer“ Werte muss die Annahme halten, dass die Angaben der Eltern unverfälscht sind und somit als Referenzwert dienen können.

Nach Maaz et al. (2006) können 15-Jährige in Deutschland von den untersuchten Merkmalen der sozialen Herkunft am besten die Schulbildung der Eltern angeben vermutlich, weil sie mit dem Schulsystem selbst gut vertraut sind (Auf Basis von PISA-Daten aus dem Jahr 2000). In Bezug auf Abschlüsse mit klarer Korrespondenz zu verbreiteten Schularten - Hauptschule, Realschule und Gymnasium - ist die faktische Übereinstimmung - gemessen mittels Prozentualer Übereinstimmung (PÜ) und Cohens Kappa - am höchsten. Etwas weniger treffsicher sind ihre Angaben zum Beruf der Eltern (ISCO-kodiert). Immerhin fallen die Übereinstimmungen hier noch etwas höher aus als bei denen zur beruflichen Ausbildung. Als Erklärung wird auf die Salienz verwiesen: Die schulische Bildung stellt eine den SuS aus ihrem eigenen Leben vertraute Kategorie dar, der ausgeübte Beruf der Eltern spielt im Alltag eine größere Rolle als die berufliche Ausbildung, die eine den SuS weitgehend unbekannte Kategorie darstellt und im eigenen Lebenslauf erst noch bevorsteht (vgl. Maaz et al. 2006, S. 38 ff.).

Unter Verwendung von PISA-Daten der Jahre 2006 und 2009 aus mehreren Ländern zeigen Jerrim und Micklewright (2014), dass SuS häufiger mit den Eltern übereinstimmende Angaben zum Beruf als zum Bildungsabschluss des Vaters liefern, wobei hier nicht zwischen schulischer und beruflicher Bildung unterschieden wird - dies könnte für die Unterschiede zu den Ergebnissen bei Maaz et al. (2006) verantwortlich sein. Zudem vergleichen sie mit PIRLS-Daten der Jahre 2001 und 2006 Eltern- und SuS-Berichte zur Anzahl der Bücher im Haushalt in verschiedenen Ländern. Hier sind die Übereinstimmungen sehr gering (vgl. Jerrim und Micklewright 2014, S. 772f.).

Obschon die Daten des NEPS insgesamt und insbesondere jene der in diesem Beitrag betrachteten Startkohorte sowohl für die empirische Bildungsforschung (vgl. z. B. die aktuellen Arbeiten von Flohr et al. 2020; Guill et al. 2020; Lindemann und Gangl 2019; Miyamoto et al. 2020) als auch für die Bildungsberichterstattung von großer Bedeutung sind (Autorengruppe Bildungsberichterstattung 2020, S. XIIf., $164 \mathrm{ff}$.), ist uns bisher nur eine Studie zur Qualität der SuS-Angaben zur sozialen Herkunft für diese Datenbasis bekannt (Nusser et al. 2016). In der 9. Jahrgangsstufe an Regelschulen betragen die Kappa-Werte zur Übereinstimmung der Bildungsangaben 0,53 und 0,47 bei Müttern und Vätern. Im Rahmen einer Machbarkeitsstudie wurden in NEPS auch Kinder und Jugendliche in Förderschulen mit dem Schwerpunkt Lernen befragt. Hier fallen in der gleichen Jahrgangsstufe die Werte mit 0,29 und 0,25 deutlich geringer aus (Nusser et al. 2016, S. 260). Doch auch die KappaWerte der SuS an Regelschulen deuten nach der Klassifikation von Wirtz und Caspar nicht auf eine gute Übereinstimmung, die sie erst ab einem Kappa von 0,6 als gegeben sehen (vgl. Wirtz und Caspar 2002, S. 59).

Lien et al. (2001, S. 733 ff.) berichten für norwegische SuS im Alter von 13 und 15 Jahren sehr hohe Übereinstimmungsraten bei Angaben zum Beruf der Eltern. 
Die Kappa-Werte betragen 0,65-0,86 für Mütter und Väter. Bei den Bildungsabschlüssen liegen die Kappa-Werte nur bei 0,38 (Mutter) und 0,30 (Vater). In einer Studie von Wittrock et al. (2017) mit Jugendlichen aus Katar fällt die Übereinstimmung der Angaben zu den Bildungsabschlüssen mit einem Kappa von 0,58 moderat aus. Nach Ensminger et al. (2000, S. 403 ff.) stimmen hingegen die Angaben von 10 bis 19-jährigen SuS in den USA zu den Bildungsabschlüssen der Eltern häufig mit deren eigenen Angaben überein. ${ }^{1}$ Eingeschränkt werden diese Befunde jedoch durch den hohen Item-Nonresponse der SuS, insbesondere bei Angaben zu Vätern (vgl. Ensminger et al. 2000, S. 403 ff.). Nach Engzell und Jonsson (2015) sind die Übereinstimmungen der Angaben von 14- und 15-Jährigen in Deutschland, England, den Niederlanden und Schweden im Children of Immigrants Longitudinal Survey in Four European Countries (CILS4EU) zu den Bildungsabschlüssen nicht besonders hoch (Korrelationen von 0,37-0,61), für Angaben zum Beruf etwas höher (Korrelationen von 0,47-0,75). Sie sehen einen Trade-off zwischen einer Verringerung der auswertbaren Fallzahlen durch einen hohen Unit-Nonresponse der Eltern auf der einen und einer mäßigen Qualität der SuS-Angaben auf der anderen Seite (Engzell und Jonsson 2015, S. $316 \mathrm{ff}$.).

Bezogen auf Übereinstimmungen lässt sich festhalten, dass die Angaben der SuS zum Beruf der Eltern in den meisten Studien als vertretbar bewertet werden, die Angaben zu den Bildungsabschlüssen der Eltern hingegen oft nicht - die meisten Studien betrachten hierbei die schulische und berufliche Bildung zusammen. In der Bewertung dieser Ergebnisse muss jedoch auch die variierende Methodik beachtet werden. Nach einer ersten Übersicht zum Ausmaß von Übereinstimmungen in SuSund Elternangaben werden im Folgenden ausgewählte systematische Zusammenhänge beleuchtet.

\subsection{Bisherige Befunde zu systematischen Einflussfaktoren auf korrekte SuS- Angaben zur sozialen Herkunft}

Kinder, die mit ihren Eltern zusammen Mahlzeiten einnehmen und viel mit ihnen kommunizieren, begehen seltener Fehler (vgl. Kreuter et al. 2006, S. 3471 ff., 2010, S. 131f.). Vergleichbare Befunde liefern Ridolfo und Maitland (2011, S. 99 ff.) für die USA, wonach auch hier engere Beziehungen zwischen Kind und Mutter mit höheren Übereinstimmungsraten bei den Bildungsabschlüssen der Mutter einhergehen.

In einer logistischen Regression der Übereinstimmung auf individuelle und sozioökonomische Faktoren zeigt sich, dass bessere Schulnoten und höhere Bildungsabschlüsse der Eltern (Elternangabe) die Chance einer Übereinstimmung signifikant erhöhen (vgl. Wittrock et al. 2017, S. 741 ff.). An Gymnasien werden im Vergleich zu anderen Schularten die höchsten Übereinstimmungsraten berichtet (vgl. Kreuter et al. 2006, S. 3471 ff., 2010, S. 131 f.). Besonders niedrige Quoten werden für SuS in

\footnotetext{
1 Auch nach West et al. (2001) liefern SuS valide Angaben zum Beruf der Eltern. Ihre Studie basiert auf Angaben von Elfjährigen aus der West of Scotland Study, in der Fragebogen mit anschließenden Kurzinterviews kombiniert wurden. Aufgrund der kombinierten Erhebungsmethode sind die Schlüsse jedoch nicht auf vollständig standardisierte Befragungen übertragbar. Eher sind die angewendeten Methoden als Möglichkeit anzusehen, die Validität von SuS-Daten zu steigern (2001, S. 542 ff., 555 ff.).
} 
Förderschulen berichtet. Hier fallen die Werte für Cohens Kappa deutlich niedriger als an Regelschulen aus (0,25 bis 0,29 vgl. mit 0,47 bis 0,53 , s. oben) und zusätzlich ist der Item-Nonresponse ca. dreimal so hoch wie in Regelschulen (24,0\% vs. 63, $1 \%$ Item-Nonrespone bei Bildungsangaben zu Müttern, 20,0\% zu 70,8\% bei Angaben zu Vätern; Nusser et al. 2016, S. 261). Dieser Gruppenvergleich unterstreicht die Bedeutung kognitiver Fähigkeiten zur Beantwortung von Fragen.

Kreuter et al. zeigen, dass SuS, die signifikant bessere Testleistungen in Mathematik erreichen, auch häufiger gleiche Angaben wie ihre Eltern liefern. Dies gilt auch unter Kontrolle der besuchten Schulart (Kreuter et al. 2006, S. $3471 \mathrm{ff}$., 2010, S. $131 \mathrm{f}$.). Auch in den Analysen von Jerrim und Micklewright weisen SuS, deren Angaben mit denen der Eltern übereinstimmen, höhere Testleistungen auf (2014, S. 773 ff.). Nur Emsinger et al. (2000) beobachten keine Zusammenhänge zwischen den schulischen Leistungen und der Übereinstimmung von SuS- und Elternangaben. $\mathrm{SuS}$, die besser in der Schule sind, weisen bei ihnen etwas seltener Nonresponse auf (Ensminger et al. 2000, S. 405 ff.). Ridolfo und Maitland (2011, S. 98 ff.) sowie Wittrock et al. (2017, S. $741 \mathrm{ff}$.) berichten, dass mit dem Alter der SuS die Übereinstimmungsraten steigen. Vermutlich beruhen die Alterseffekte ebenfalls auf kognitiven Fähigkeiten.

Es kann festgehalten werden, dass die Übereinstimmungen in den Angaben systematisch nach Merkmalen der SuS, z. T. auch nach Merkmalen der Eltern differieren. Höhere kognitive Fähigkeiten, der Besuch einer anspruchsvolleren Schulform, vermehrte Kommunikation mit den Eltern sowie Bildungsabschlüsse der Eltern, die für die SuS bekannte Kategorien darstellen, gehen mit höheren Übereinstimmungsraten einher.

Die bisherigen Befunde basieren (meist) auf dem Vorliegen von ,auswertbaren“ Angaben der SuS. (Item-)Nonresponse wird zwar berichtet (z. B. Engzell und Jonsson 2015; Jerrim und Micklewright 2014; Nusser et al. 2016), nicht aber in die Analysen korrekter Angaben einbezogen. Das Verweigern einer Antwort wie auch das Liefern nicht kodierbarer Antworten sind mögliche Ergebnisse des Prozesses des Antwortens in standardisierten Umfragen und somit als ,falsche“ Angaben zu den Bildungsabschlüssen oder zur beruflichen Tätigkeit der Eltern zu werten.

\subsection{Bisherige Befunde zu möglichen Verzerrungen bei der Verwendung von SuS-Angaben bei der Untersuchung von Bildungsungleichheiten}

Mit einer Regression der Testleistungen in Mathematik auf den SES zeigen Kreuter et al. (2010, S. $135 \mathrm{ff}$.), dass der Steigungskoeffizient für die berufliche Bildung des Vaters bei Nutzung von Elternangaben höher ausfällt als bei Nutzung von SuSAngaben. Jerrim und Micklewright regressieren die Lesekompetenz auf SuS- und Eltern-Angaben zur sozialen Herkunft. Sie schließen aus ihren Analysen, dass im Fall der Bildung des Vaters die Nutzung von SuS-Angaben zu verzerrten Schätzern führt. Der SES-Gradient fällt bei Nutzung von SuS-Angaben geringer aus. Im Fall des Berufs des Vaters sind die Ergebnisse jedoch gegenüber der Nutzung von SuSAngaben robust (Jerrim und Micklewright 2014, S. 773 ff.).

Engzell und Jonsson (2015) liefern Hinweise, dass auch die Verwendung von elterlichen Angaben zum SES zu einer Unterschätzung des Zusammenhangs zwi- 
schen SES und Testleistung führen kann, weil sowohl geringe Testleistungen des Kindes als auch niedriger SES mit geringeren Teilnahmewahrscheinlichkeiten der Eltern einhergehen (Engzell und Jonsson 2015, S. 321).

\subsection{Kognitive Theorie des Antwortverhaltens}

Mittels der kognitiven Theorie des Antwortverhaltens kann der Prozess des Beantwortens von standardisierten Fragen näher beleuchtet werden. Sie unterteilt diesen in vier Phasen: (1) das Verständnis der Frage, (2) die Suche nach relevanten Informationen und Erinnerungen, (3) die Bewertung der gefundenen Informationen sowie (4) das Antworten selbst. In jeder dieser Phasen können spezifische Herausforderungen zu Messfehlern führen (vgl. Tourangeau et al. 2000, S. 7 ff., 13 ff., 77, 255 ff.; Vanette und Krosnick 2014, S. 313).

In der Verständnis-Phase entstehen Fehler meist, wenn die Frage falsch verstanden oder interpretiert wird. Es ist also wichtig, Konzepte abzufragen, die allen Befragten verständlich sind. In der zweiten Phase suchen Befragungspersonen nach Wissen, das zur Beantwortung der Frage relevant ist. Je leichter abrufbar Informationen sind, desto weniger aufwändig sollte die Suche nach ihnen sein. Der Abruf einer Erinnerung oder Information kann sukzessive vernetzte Erinnerungen und Informationen aktivieren. Nun werden die aktivierten Informationen in eine kohärente Bewertung der Frage integriert. In der letzten Phase wird anhand der erstellten Bewertung eine Antwort selektiert bzw. bei offenen Fragen eine Antwort gebildet. Je weniger trennscharf die vorgegebenen Antwortkategorien sind, desto größerer ist das Risiko von Messfehlern. Möglich ist auch, dass aufgrund sozialer Erwünschtheit in den Phasen drei und vier eine abweichende Antwort gewählt wird (vgl. Tourangeau et al. 2000, S. 7 ff., 13 ff., 77, 255 ff.; Vanette und Krosnick 2014, S. 313).

Die Theorie geht davon aus, dass die gesuchte Information mental gespeichert ist. Sollte den SuS die Information, welchen Bildungsabschluss ihre Eltern haben, aber tatsächlich völlig unbekannt sein, könnten sie die Antwort verweigern oder willkürlich antworten. Weiter geht die Theorie davon aus, dass Selbsterlebtes in detaillierterer und lebendigerer Form gespeichert wird und Teil des autobiografischen Narrativs wird. Daher postulieren Tourangeau et al., dass Selbsterlebtes akkurater wiedergegeben wird als Informationen aus zweiter Hand (vgl. Tourangeau et al. 2000, S. 15 ff., 65 f.; Vanette und Krosnick 2014, S. 313 f.).

Da die korrekte Abfolge dieser vier Schritte mitunter große kognitive Ressourcen erfordert, müssen die Befragten gewillt sein, diese zu investieren. Sind sie es nicht, kann es nach Vanette und Krosnick (2014) zum sogenannten Satisficing kommen. In der schwachen Variante verkürzen Befragte die Phasen zwei und drei und suchen nur unvollständig nach Informationen, bzw. bewerten sie nur oberflächlich. Im starken Satisficing suchen die Befragten gar nicht mehr nach Informationen, sondern wählen zufällig oder intuitiv eine Antwort aus. Das vollständige und korrekte Durchlaufen der vier Antwortphasen wird hingegen als Optimizing bezeichnet (Vanette und Krosnick 2014, S. 314f.).

In der Methodenliteratur werden auch die Begriffe, ,inattentive or careless responses“ (Meade und Craig 2012, S. 437 ff.) sowie ,insufficient effort (...) responding“ (Curran 2016, S. 4 f.) verwendet. Aufgrund mangelnder Motivation oder Ermüdung 
werden kaum oder nur wenig kognitive Ressourcen zur Beantwortung der Frage aufgebracht. Im Extremfall werden (bei geschlossenen Fragen) willkürlich Kreuze gesetzt (Meade und Craig 2012, S. 437 ff.). Der Unterschied zur kognitiven Theorie des Antwortverhaltens ist gradueller Natur. In der Forschung zu unachtsamen Antwortverhalten wird stärker thematisiert, dass bereits die Frage gar nicht oder nicht aufmerksam gelesen wird, dass also nicht nur die Suche nach Informationen abgekürzt wird (Curran et al. 2016, S. $13 \mathrm{ff}$.). Eine starke Form des Satisficings stellt das Auslassen einer Antwort oder das Ankreuzen einer „Weiß nicht“-Option dar (Vanette und Krosnick 2014, S. 315 f.).

In verschiedenen Studien wurde die Wirkung von Persönlichkeitsmerkmalen auf Schulleistungen und auf beruflichen Erfolg untersucht. In diesen Studien stand Gewissenhaftigkeit - welche den Willen umfasst, Aufgaben bestmöglich zu erfüllen und Verpflichtungen nachzukommen, und eine offensichtliche Nähe zu Optimizing aufweist - in statistisch signifikant positiven Zusammenhängen mit Bildungs- und Berufserfolg (vgl. Barrick und Mount 1991; De Feyter et al. 2012; Vedel 2014). Befragte mit höherem Ausmaß an Gewissenhaftigkeit und sozialer Verträglichkeit gehören auch seltener zu jenen Befragten, die ,unachtsames“ Antwortverhalten an den Tag legen (Maniaci und Rogge 2014, S. 71 f.; Meade und Craig 2012, S. 446). Zusammenfassend lässt sich festhalten, dass ausgeprägtere Gewissenhaftigkeit, stärkere kognitive Fähigkeiten, größere Lesefähigkeiten und leichter abrufbare relevante Informationen das vollständige Durchlaufen der vier Phasen fördern sollten.

\subsection{Hypothesen und Forschungsfrage}

Vor dem Hintergrund der theoretischen Überlegungen und des Forschungsstands werden Hypothesen hergeleitet. Als Indikator zur sozialen Herkunft verwenden wir den Bildungsstand der Eltern, weil dieser im Gegensatz zur beruflichen Tätigkeit stabiler ist und zugleich in den Erklärungen von Bildungsungleichheiten eine zentrale Rolle einnimmt (Bradbury et al. 2015, S. 25; Erikson 2019, S. 41 ff.). Außerdem werden hier häufig stärkere Dissonanzen zwischen SuS und Eltern berichtet (vgl. Jerrim und Micklewright 2014). Offene Angaben zur beruflichen Tätigkeit müssen zudem codiert werden, was eine zusätzliche Quelle für Abweichungen in ElternKind-Angaben darstellen könnte. Wenn im Folgenden von Phase 1, 2 oder 3 die Rede ist, beziehen diese sich auf die Phasen der kognitiven Theorie des Antwortverhaltens aus Abschn. 2.4.

Der Prozess des Antwortens setzt voraus, dass SuS die Frage richtig verstehen und interpretieren (Phase 1), Sprach- und Lesefähigkeiten sollten hierfür zentral sein. Hypothese 1 (H1): Jugendliche mit besseren Sprachfähigkeiten liefern häufiger Angaben, die mit denen ihrer Eltern übereinstimmen.

Den SuS sind die Bildungsabschlüsse ihrer Eltern nicht so präsent wie diesen selbst, da die Eltern sie selbst „erworben“ haben, diese zu verschiedenen Gelegenheiten wie Bewerbungsschreiben wiederholt abgerufen haben und ihnen die Verknüpfungen der Frage mit den mental gespeicherten Informationen (Phase 2 und 3) leichter fallen sollte. Da im NEPS nur ein Elternteil befragt wird, gibt es, sofern ein weiteres Elternteil im Haushalt lebt, auch auf Seiten der Eltern Proxy-Angaben zu den Bildungsabschlüssen des anderen Elternteils. H2: Der Zusammenhang zwischen 
den Angaben der Jugendlichen und den Eltern fallen für das Elternteil, das befragt wurde, enger aus als zu dem Elternteil, über das berichtet wurde.

Den Jugendlichen mag das Bildungssystem, das ihre Eltern im Ausland durchlaufen haben, weitestgehend unbekannt sein und somit auch die Äquivalenz zu in Deutschland erwerbbaren Abschlüssen. Daher sollte die Erinnerung von im Ausland erworbener Abschlüsse und deren Zuordnung zu den deutschen Bildungsabschlüssen in Phase 2 und 3 schwerer fallen. H3: Jugendliche, deren Eltern im Ausland einen ausländischen Bildungsabschluss besitzen, haben niedrigere Übereinstimmungsraten.

Das Auffinden von mentalen Repräsentationen sollte leichter und akkurater sein, je stärker die Repräsentationen verankert sind. Jugendliche, die mehr mit ihren Eltern kommunizieren oder für die das Thema unabhängig von der aktuellen Befragung relevant ist, sollten besser verankerte Informationen zu den Bildungsabschlüssen der Eltern besitzen. Daher sollte es ihnen leichter fallen, in Phase 2 und 3 die relevanten Informationen zu finden. H4: Jugendliche, die mehr mit ihren Eltern kommunizieren, haben höhere Übereinstimmungsraten.

Jugendliche mit hoher Gewissenhaftigkeit investieren in Phase 2 und 3 eher die Zeit und kognitiven Ressourcen zur optimalen Beantwortung einer Frage (Optimizing statt Satisficing oder unachtsames Antworten). H5: Je höher die Gewissenhaftigkeit eines Jugendlichen, desto häufiger stimmen die Angaben mit denen der Eltern überein.

Die Hypothesen $\mathrm{H} 1$ bis $\mathrm{H} 4$ beziehen sich primär auf das Vermögen, die richtige Antwort zu finden, H5 hingegen auf die Motivation, kognitive Ressourcen aufzuwenden. Wie weiter oben besprochen, erfordern steigende kognitive Kosten bei der Beantwortung einer Frage eine höhere Motivation. Hier sind also auch wechselseitige Beeinflussungen denkbar.

Bisherige Forschung hat gezeigt, dass die Zusammenhänge zwischen sozialer Herkunft und Testleistungen schwächer ausfallen, wenn Angaben der SuS genutzt werden. Analog zu Kreuter et al. (2010) wird die Frage, ob und wie stark mögliche Verzerrungen sind, am Beispiel der Mathematikleistungen untersucht. Forschungsfrage 1: Verzerrt die Nutzung von SuS-Angaben zur sozialen Herkunft das Ausmaß von Bildungsungleichheiten?

\section{Daten und Methoden}

Die Hypothesen und Forschungsfragen werden mit Daten der Startkohorte 4 (SC4) des Nationalen Bildungspanels (NEPS) analysiert (Blossfeld und Roßbach 2019; https://doi.org/10.5157/NEPS:SC4:10.0.0). ${ }^{2}$ Die SC4 ist eine Panelbefragung, die

\footnotetext{
2 „Diese Arbeit nutzt Daten des Nationalen Bildungspanels (NEPS): Startkohorte Klasse 9, https://doi. org/10.5157/NEPS:SC4:10.0.0. Die Daten des NEPS wurden von 2008 bis 2013 als Teil des Rahmenprogramms zur Förderung der empirischen Bildungsforschung erhoben, welches vom Bundesministerium für Bildung und Forschung (BMBF) finanziert wurde. Seit 2014 wird NEPS vom Leibniz-Institut für Bildungsverläufe e. V. (LIfBi) an der Otto-Friedrich-Universität Bamberg in Kooperation mit einem deutschlandweiten Netzwerk weitergeführt." https://www.neps-data.de/de-de/datenzentrum/datenunddokumentation/ startkohorteklasse9/datenundzitation.aspx (zuletzt aufgerufen 20.01.2020).
} 
2010 in der neunten Klasse startete und Daten zur Entwicklung von Kompetenzen, zu Bildungsverläufen und Arbeitsmarkteinstiegsprozessen bereitstellt. Wir verwenden Daten aus der ersten Erhebungswelle, in der die SuS getestet und schriftlich, ihre Eltern telefonisch befragt wurden. An der ersten Welle nahmen 15.239 SuS in Regelschulen und $1186 \mathrm{SuS}$ in Förderschulen teil (IEA DPC o.J.a, S. 22, IEA DPC o.J.b, S. 18).

Die SuS werden gefragt: ,Welchen höchsten Bildungsabschluss haben deine Eltern?“ Diese Frage ist mit folgenden Ausfüllhinweisen versehen: „Falls deine Eltern ihren Schulabschluss nicht in Deutschland gemacht haben, nenne bitte den entsprechenden deutschen Schulabschluss. Zutreffendes bitte für Vater und Mutter ankreuzen“ (NEPS 2013, S. 14, 145, Herv. im Original unterstrichen). Als Antwortkategorien steht ihnen zur Verfügung: „keinen Schulabschluss“, „Haupt-/ Volksschulabschluss, 8. Klasse POS“, „Mittlere Reife/Realschulabschluss, 10. Klasse POS“, „Fachabitur, Abitur, 12. Klasse EOS“, „Fachhochschulabschluss, Hochschulabschluss“, „Promotion (Doktortitel)“, ,, anderen Abschluss“, „weiß nicht“. In der telefonischen Elternbefragung erfolgt die Abfrage der Bildungsabschlüsse deutlich differenzierter (NEPS 2013, S. $248 \mathrm{ff}$, $274 \mathrm{ff}$.). Diese Angaben werden in das Kategorienschema überführt, das den SuS vorgelegt wurde, wobei wir Promotionen der Kategorie Hochschulabschlüsse zuordnen. Sonstige Abschlüsse wurden ausgeschlossen. In einem nächsten Schritt wird die kategoriale Variable „Übereinstimmung“ (0), „keine Übereinstimmung“ (1) und keine SuS-Angabe (2) zum Abschluss der Mutter und des Vaters respektive gebildet. Haben die Eltern keine Angaben geliefert, enthält die Variable Fehlwerte.

In der Elternbefragung soll die Befragungsperson sowohl zu sich selbst als auch zu der im Haushalt gemeinsam lebenden Partnerin oder Partner Angaben tätigen. In beiden Fällen kann es sich somit z. B. auch um Stiefeltern handeln. Angaben zu Elternteilen, in denen sich SuS und das antwortende Elternteil auf unterschiedliche Personen beziehen, werden ausgeschlossen. Würden wir dies nicht tun, bestünde die Gefahr, dass Inkonsistenzen daher rühren, dass ein Jugendlicher z. B. Angaben zum leiblichen Vater, die Mutter jedoch zum Stiefvater liefert.

Da die Lesekompetenzen erst in Welle 2 erhoben wurden, greifen wir auf die in Welle 1 erfolgte Testung zum rezeptiven Wortschatz zurück. Hier kommt im NEPS eine deutschsprachige Variante des Peabody Picture Vocabulary Tests (PPVT) nach Dunn und Dunn (2004) zum Einsatz (NEPS 2018, S. 10). Die Wortschatzkenntnisse werden in Quartile unterteilt (Hypothese 1). Im Elternfragebogen wird erhoben, in welcher Beziehung das Auskunft gebende Elternteil und seine/ihre Partner/in zum Zielkind stehen. Anhand dieser Informationen wird eine Dummy-Variable erstellt, die erfasst, ob die (Stief-/Adoptiv-)Mutter (einschließlich Pflegemutter, Freundin des Vaters $=1$ ) oder der (Stief-/Adoptiv-)Vater (einschließlich Pflegevater, Freund der Mutter =0) antwortet (Hypothese 2). Zudem wird auf die Information zurückgegriffen, ob die Eltern ihren höchsten Bildungsabschluss im Ausland (= 1, sonst 0) erworben haben (Hypothese 3). Die Kommunikation im Elternhaus wird mit einem Index basierend auf vier Antworten zu Diskussionen im Elternhaus über Bücher, 
Tab. 1 Ausschluss von Fällen in den vier Analysestichproben

\begin{tabular}{|c|c|c|c|c|}
\hline \multirow[b]{2}{*}{ Grund für Ausschluss } & \multicolumn{2}{|c|}{$\begin{array}{l}\text { Stichproben zur Vorhersage } \\
\text { von SuS-Antworten }\end{array}$} & \multicolumn{2}{|c|}{$\begin{array}{l}\text { Stichproben zum Ausmaß sozia- } \\
\text { ler Ungleichheiten in Testleis- } \\
\text { tungen }\end{array}$} \\
\hline & Mutter & Vater & Mutter & Vater \\
\hline Fallzahl (brutto) & 16.425 & 16.425 & 16.425 & 16.425 \\
\hline SuS in Förderschulen & -1186 & -1186 & -1186 & -1186 \\
\hline $\begin{array}{l}\text { SuS hat in Welle } 1 \text { nicht teilge- } \\
\text { nommen }\end{array}$ & -749 & -749 & -749 & -749 \\
\hline $\begin{array}{l}\text { Keine Information von Eltern zu } \\
\text { ihren Bildungsabschlüssen }\end{array}$ & -6558 & -8311 & -6558 & -8311 \\
\hline Unterschiedliche Elterndefinition & -147 & -314 & -147 & -314 \\
\hline $\begin{array}{l}\text { SuS liefert keine Angabe zum } \\
\text { Bildungsabschluss der Eltern }\end{array}$ & n.r. & n.r. & -1644 & -1471 \\
\hline Ergebnis aus Wortschatztest fehlt & -9 & -4 & n. r. & n. r. \\
\hline Ergebnis aus Mathematiktest fehlt & n.r. & n.r. & -4 & -1 \\
\hline $\begin{array}{l}\text { Land des Bildungsabschlusses } \\
\text { unbekannt }\end{array}$ & -0 & -2 & n. r. & n. r. \\
\hline Fallzahl (netto) & 7776 & 5859 & 6137 & 4393 \\
\hline Anzahl Schulen & 544 & 536 & 536 & 519 \\
\hline
\end{tabular}

Quellen: https://doi.org/10.5157/NEPS:SC4:10.0.0; eigene Berechnungen

SuS Schülerinnen und Schüler, n.r. hier nicht relevant

Filme, Politik und Kunst operationalisiert (Hypothese 4) (Cronbachs alpha=0,64). ${ }^{3}$ Zur Erfassung von Persönlichkeitseigenschaften wird im NEPS eine Ultra-Kurzskala von Rammstedt und John (2005) eingesetzt. Gewissenhaftigkeit wird hier mit dem Summenscore der beiden Items „Ich bin bequem, neige zur Faulheit“ und „Ich erledige Aufgaben gründlich“ und einer fünfstufigen Antwortskala von „trifft gar nicht zu“ bis „trifft völlig zu“ (NEPS 2013, S. 11, Frage 10c, 10h) gemessen (Cronbachs alpha $=0,53)$ (Hypothese 5). Auch hier werden wieder Quartile gebildet, wobei aufgrund der wenigen Ausprägungen der diskreten Verteilung die Quartile unterschiedlich stark besetzt sind.

Zur Frage, ob Bildungsungleichheiten unterschätzt werden, wenn anstelle von Eltern- auf SuS-Angaben zurïckgegriffen wird, werden - wie auch bei Kreuter et al. (2010) - die Mathematikleistungen als abhängige Variable verwendet. Zur Erfassung derselben kam in der ersten Welle der neunten Jahrgangsstufe ein eigens entwickelter Test zum Einsatz. Die Ergebnisse aus diesem Test werden von NEPS Rasch-skaliert, so dass latente Personenfähigkeitsparameter in den Daten vorliegen (Duchhardt und Gerdes 2013). Die unabhängige Variable (soziale Herkunft) bezieht sich auf den Bildungsabschluss und umfasst die bereits oben beschriebenen fünf Kategorien von „kein Schulabschluss“ bis hin zu „Hochschulabschluss“. Diese fünf Kategorien werden mit je einer Dummy-Variable erfasst.

\footnotetext{
3 „Wie oft kommt es im Allgemeinen vor, dass du mit deiner Mutter oder deinem Vater (...) [ ] nie oder selten [ ] mehrmals im Monat [ ] einmal in der Woche [ ] mehrmals in der Woche [ ] täglich a) ... über Bücher redest? b) ... über Filme oder Fernsehsendungen redest? c) ... über politische oder soziale Fragen redest? d) ... über Kunstwerke oder Kunst im Allgemeinen redest?“ (NEPS 2013, S. 30, Frage 40).
} 
Tab. 1 zeigt Gründe und Ausmaß von Fallausschlüssen nach Mutter und Vater getrennt. Die SuS in Förderschulen werden ausgeschlossen, weil bei ihnen Variablen, die für die hier vorgelegten Analysen zentral sind, nicht erhoben wurden. Somit beziehen sich die Analysen ausschließlich auf Jugendliche in Regelschulen. Von den SuS in Regelschulen haben 749 in der ersten Welle nicht teilgenommen (,,temporärer Ausfall“). Bei den nun verbleibenden Fällen fehlen zu 6558 Müttern und zu 8311 Vätern Angaben. In der Mehrzahl der Fälle $(n=6206)$ handelt es sich um „Partial-unit-nonresponse“, die Nicht-Teilnahme der Eltern an der ersten Elternbefragung. ${ }^{4}$ Die restlichen Fehlwerte kommen durch Antwortverweigerung, nicht verwertbare Angaben oder fehlende Elternteile im Haushalt zustande. Von den verbleibenden Fällen werden weitere ausgeschlossen, weil SuS sich auf ein anderes Elternteil beziehen (z.B. leiblicher Vater statt im gemeinsamen Haushalt lebender Stiefvater) (bei Müttern 78 Fälle, bei Vätern 210 Fälle), weil SuS angeben, (Halb-)Waise zu sein oder ihren Vater nicht zu kennen (bei Müttern 11 Fälle, bei Vätern 33 Fälle), weil SuS trotz anderslautender Ausfüllhinweise bei Mutter oder Vater mehrere Antwortoptionen oder auch gar keine Person ankreuzen (bei Müttern 58 Fälle, bei Vätern 71 Fälle).

Fehlende Werte in Kovariaten werden in der multinomialen logistischen Regression mithilfe einer eigenen Dummy-Variablen erfasst. Im Fall von fehlenden Ergebnissen aus dem Wortschatztest und fehlender Information, ob die Mutter oder der Vater den Bildungsabschluss im Ausland erworben hat, ist dies aufgrund der geringen Anzahl an Fehlwerten nicht möglich. Sie werden deshalb ausgeschlossen.

Für die Analysen der SuS-Angaben zum Bildungsabschluss der Eltern verbleiben somit 7776 Fälle bei Angaben zum Bildungsabschluss der Mutter und 5859 Fälle bei denen zum Vater. Die SuS sind 15,1 bzw. 15,0 Jahre (Standardabweichung 0,6 in beiden Analysestichproben); fast die Hälfte ist weiblich (49,6 bzw. 48,8\%) (soziodemographische Kennwerte nicht tabellarisch ausgewiesen). Die SuS stammen aus 544 bzw. 536 Schulen.

Zur Bearbeitung der Forschungsfrage, ob und wie sich das Ausmaß der sozialen Herkunft unterscheidet, wenn SuS- anstelle von Elternangaben verwendet werden, sind die ersten Schritte des Fallausschlusses identisch zu den zuvor berichteten. Hier kommt aber hinzu, dass wir uns auf Eltern-Kind-Paare beschränken, bei denen Informationen von beiden Seiten vorliegen. In der Stichprobe zu Müttern werden 1644 und in der zu Vätern 1471 Fälle ausgeschlossen, weil Jugendliche keine Angabe zum Bildungsabschluss der Eltern geliefert haben (siehe Tab. 1, die beiden letzten Spalten). In den nun verbleibenden Stichproben fehlen zu 4 bzw. 1 SuS Informa-

\footnotetext{
${ }^{4}$ Die Beschränkung auf Fälle mit gültiger Elternangabe reduziert nicht nur die Stichprobe, sondern die Teilnahme der Eltern ist auch selektiv. Haben die Jugendlichen einen Migrationshintergrund, besuchen sie eine andere Schulart als das Gymnasium, sind sie etwas älter im Vergleich zu ihren Mit-SuS und handelt es sich um Mädchen, nehmen Eltern seltener teil (Steinhauer und Zinn 2016, S. 8 f., 19). Um diesen selektiven Nonresponse zu korrigieren, stellt das NEPS-Datenzentrum Gewichte bereit, die zudem Informationen zum Ziehungsdesign und zur SuS-Teilnahme enthalten (Steinhauer und Zinn 2016). Siehe zum Umgang mit selektiven Ausfällen auch Fußnote 6.
} 
tionen zur Mathematikkompetenz, der abhängigen Variable. Somit liegen für 6137 Mutter-Kind- und für 4393 Vater-Kind-Paare auswertbare Daten vor. ${ }^{5}$

Zur Testung der Hypothesen werden multinomiale logistische Regressionen geschätzt, da die abhängige Variable drei Ausprägungen hat. Zusätzlich zu den relativen Risiken werden marginale Effekte ausgewiesen, da bei multinomialen Logit-Modellen positive Logit-Koeffizienten bzw. exponierte Schätzkoeffizienten, die größer als 1 sind, nicht wie bei binären logistischen Regression zwangsläufig mit höheren Wahrscheinlichkeiten einhergehen. Bei den berechneten und graphisch dargestellten marginalen Effekten handelt es sich um Differenzen in den Wahrscheinlichkeiten, dass eine spezifische Ausprägung der abhängigen Variable bei Vorliegen und NichtVorliegen eines Merkmals beobachtet wird (StataCorp 2017, S. $1434 \mathrm{ff} ., 1516 \mathrm{ff}$.). Da in NEPS SC4 zunächst Schulen und dann SuS gezogen wurden, und SuS ein und derselben Schule sich möglicherweise ähnlicher als zwei zufällig gezogene Neuntklässler/innen sind, werden die Standardfehler mit dem Sandwich-Huber-Verfahren korrigiert (cluster-Option in Stata; StataCorp 2017, S. 323 ff.).

Zur Beantwortung von Forschungsfrage 1 kommen lineare Regressionsmodelle zum Einsatz. Hier steht der Vergleich von Regressionskoeffizienten zweier getrennter Modellschätzungen im Fokus. Zur Prüfung, ob zwei Regressionskoeffizienten statisch signifikant voneinander verschieden sind, wird in der Literatur häufig ein gepoolter t-Test angewandt, der auf Unterschiede in einer Maßzahl zwischen zwei Gruppen testet (vgl. Clogg et al. 1995; Paternoster et al. 1998). Diesem Test liegt die Annahme zugrunde, dass keine Kovarianz zwischen den beiden Koeffizienten vorliegt. Da es sich bei den Regressionen zu Forschungsfrage 1 jedoch um Angaben zum selben Konzept (Bildungsabschluss der Eltern) bei den gleichen Untersuchungseinheiten (Familien, bzw. Eltern und ihre Kinder) handelt, und diese eine moderate Übereinstimmung aufweisen, ist diese Annahme nicht haltbar. Nach Mize et al. (2019) führt die Ignorierung der Kovarianz zu einer Verzerrung der Standardfehler, wenn Koeffizienten mittels t-Test verglichen werden, weshalb hier die Methode „seemingly unrelated estimation (SUEST)“ mit Bootstrapping zum Einsatz kommt (Mize et al. 2019, S. 8 f., 17f.).

\section{Ergebnisse}

$52 \%$ der Jugendlichen stimmen mit der Elternangabe zum Bildungsabschluss der Mutter überein. $27 \%$ der Jugendlichen liefern eine abweichende Angabe, $21 \%$ geben keine (oder keine kodierbare) Antwort. Im Fall von Vätern sind die entsprechenden Anteile 47, 28 und 25\% (siehe Tab. 4 im Anhang). Wird die Stichprobe auf die ersten beiden Möglichkeiten beschränkt, wie im berichteten Forschungsstand üblich, betragen die prozentualen Übereinstimmungen in den Eltern-Kind-Angaben 66 und $63 \%$, Cohens Kappa 0,52 und 0,50 (siehe Tab. 5 im Anhang).

\footnotetext{
5 Das durchschnittliche Alter der SuS beträgt 15,1 bzw. 15,0 Jahre (Standardabweichung 0,6 in beiden Analysestichproben); 49,8 bzw. 48,6\% sind weiblich.
} 
Tab. 2 Multinomiale Regression zur Übereinstimmung von Eltern-Kind-Angaben zum Bildungsabschluss von Mutter und Vater; relative Risiken und robuste Standardfehler

\begin{tabular}{|c|c|c|c|c|c|c|}
\hline \multirow[b]{2}{*}{$\begin{array}{l}\text { Verhältnis der } \\
\text { SuS- zur Eltern- } \\
\text { angabe }\end{array}$} & \multicolumn{3}{|c|}{ Modell 1 (Mütter) } & \multicolumn{3}{|c|}{ Modell 2 (Väter) } \\
\hline & $\begin{array}{l}\text { Inkonsistent/ } \\
\text { konsistent }\end{array}$ & $\begin{array}{l}\text { Keine/ } \\
\text { konsistent }\end{array}$ & $\begin{array}{l}\text { Keine/in- } \\
\text { konsis- } \\
\text { tent }^{\mathrm{a}}\end{array}$ & $\begin{array}{l}\text { Inkonsistent/ } \\
\text { konsistent }\end{array}$ & $\begin{array}{l}\text { Keine/ } \\
\text { konsistent }\end{array}$ & $\begin{array}{l}\text { Keine/in- } \\
\text { konsistent }^{\mathrm{a}}\end{array}$ \\
\hline \multicolumn{7}{|c|}{ Wortschatz (Rk.: niedrigstes Quartil) } \\
\hline Zweites Quartil & $\begin{array}{l}0,860 \\
(0,067)\end{array}$ & $\begin{array}{l}0,814^{*} \\
(0,070)\end{array}$ & $\begin{array}{l}0,947 \\
(0,087)\end{array}$ & $\begin{array}{l}0,853 \\
(0,079)\end{array}$ & $\begin{array}{l}0,793^{*} \\
(0,076)\end{array}$ & $\begin{array}{l}0,930 \\
(0,091)\end{array}$ \\
\hline Drittes Quartil & $\begin{array}{l}0,842 \\
(0,075)\end{array}$ & $\begin{array}{l}0,670 * * * \\
(0,063)\end{array}$ & $\begin{array}{l}0,795^{*} \\
(0,084)\end{array}$ & $\begin{array}{l}0,841 \\
(0,082)\end{array}$ & $\begin{array}{l}0,622 * * * \\
(0,064)\end{array}$ & $\begin{array}{l}0,739 * * \\
(0,078)\end{array}$ \\
\hline Höchstes Quartil & $\begin{array}{l}0,765^{* *} \\
(0,070)\end{array}$ & $\begin{array}{l}0,667 * * * \\
(0,068)\end{array}$ & $\begin{array}{l}0,872 \\
(0,096)\end{array}$ & $\begin{array}{l}0,667 * * * \\
(0,071)\end{array}$ & $\begin{array}{l}0,616 * * * \\
(0,069)\end{array}$ & $\begin{array}{l}0,923 \\
(0,102)\end{array}$ \\
\hline \multicolumn{7}{|c|}{ Besuchte Schulform (Rk.: Hauptschule) } \\
\hline $\begin{array}{l}\text { Schule mit meh- } \\
\text { reren Bildungs- } \\
\text { gängen (SmmB, } \\
\text { ohne IGS) }\end{array}$ & $\begin{array}{l}0,692 * * \\
(0,093)\end{array}$ & $\begin{array}{l}0,906 \\
(0,149)\end{array}$ & $\begin{array}{l}1,309 \\
(0,222)\end{array}$ & $\begin{array}{l}0,661 * \\
(0,119)\end{array}$ & $\begin{array}{l}0,892 \\
(0,176)\end{array}$ & $\begin{array}{l}1,349 \\
(0,264)\end{array}$ \\
\hline Realschule & $\begin{array}{l}0,714 * * * \\
(0,067)\end{array}$ & $\begin{array}{l}0,602 * * * \\
(0,066)\end{array}$ & $\begin{array}{l}0,842 \\
(0,096)\end{array}$ & $\begin{array}{l}0,881 \\
(0,095)\end{array}$ & $\begin{array}{l}0,645^{* * * *} \\
(0,080)\end{array}$ & $\begin{array}{l}0,732 * * \\
(0,084)\end{array}$ \\
\hline $\begin{array}{l}\text { Integrierte Ge- } \\
\text { samtschule (IGS) }\end{array}$ & $\begin{array}{l}0,939 \\
(0,121)\end{array}$ & $\begin{array}{l}0,832 \\
(0,113)\end{array}$ & $\begin{array}{l}0,885 \\
(0,131)\end{array}$ & $\begin{array}{l}1,002 \\
(0,136)\end{array}$ & $\begin{array}{l}0,866 \\
(0,138)\end{array}$ & $\begin{array}{l}0,865 \\
(0,127)\end{array}$ \\
\hline Gymnasium & $\begin{array}{l}0,866 \\
(0,079)\end{array}$ & $\begin{array}{l}0,589 * * * \\
(0,058)\end{array}$ & $\begin{array}{l}0,680 * * * \\
(0,074)\end{array}$ & $\begin{array}{l}1,007 \\
(0,105)\end{array}$ & $\begin{array}{l}0,673 * * * \\
(0,077)\end{array}$ & $\begin{array}{l}0,668 * * * \\
(0,072)\end{array}$ \\
\hline \multicolumn{7}{|c|}{ Befragtes Elternteil (Rk.: Vater) } \\
\hline Mutter & $\begin{array}{l}1,062 \\
(0,082)\end{array}$ & $\begin{array}{l}0,881 \\
(0,078)\end{array}$ & $\begin{array}{l}0,829 \\
(0,083)\end{array}$ & $\begin{array}{l}0,880 \\
(0,064)\end{array}$ & $\begin{array}{l}1,031 \\
(0,081)\end{array}$ & $\begin{array}{l}1,172 \\
(0,096)\end{array}$ \\
\hline \multicolumn{7}{|c|}{ Land des höchsten Bildungsabschlusses (Rk.: Deutschland) } \\
\hline Ausland & $\begin{array}{l}2,323 * * * \\
(0,249)\end{array}$ & $\begin{array}{l}3,247 * * * \\
(0,337)\end{array}$ & $\begin{array}{l}1,398 * * \\
(0,144)\end{array}$ & $\begin{array}{l}2,064 * * * \\
(0,272)\end{array}$ & $\begin{array}{l}3,251 * * * \\
(0,386)\end{array}$ & $\begin{array}{l}1,575 * * * \\
(0,178)\end{array}$ \\
\hline \multicolumn{7}{|c|}{ Diskussionen mit Eltern (Rk.: niedrigstes Quartil) } \\
\hline Zweites Quartil & $\begin{array}{l}1,410 * * * \\
(0,107)\end{array}$ & $\begin{array}{l}0,886 \\
(0,070)\end{array}$ & $\begin{array}{l}0,628 * * * \\
(0,055)\end{array}$ & $\begin{array}{l}1,015 \\
(0,089)\end{array}$ & $\begin{array}{l}0,805^{*} \\
(0,071)\end{array}$ & $\begin{array}{l}0,793^{*} \\
(0,080)\end{array}$ \\
\hline Drittes Quartil & $\begin{array}{l}1,219^{*} \\
(0,095)\end{array}$ & $\begin{array}{l}0,776^{* *} \\
(0,067)\end{array}$ & $\begin{array}{l}0,636^{* * *} \\
(0,057)\end{array}$ & $\begin{array}{l}1,024 \\
(0,095)\end{array}$ & $\begin{array}{l}0,879 \\
(0,080)\end{array}$ & $\begin{array}{l}0,858 \\
(0,093)\end{array}$ \\
\hline Höchstes Quartil & $\begin{array}{l}1,442 * * * \\
(0,109)\end{array}$ & $\begin{array}{l}0,772 * * \\
(0,063)\end{array}$ & $\begin{array}{l}0,535^{* * *} \\
(0,050)\end{array}$ & $\begin{array}{l}1,094 \\
(0,097)\end{array}$ & $\begin{array}{l}0,699 * * * \\
(0,065)\end{array}$ & $\begin{array}{l}0,639 * * * \\
(0,060)\end{array}$ \\
\hline Fehlender Wert & $\begin{array}{l}0,809 \\
(0,278)\end{array}$ & $\begin{array}{l}2,104 * * \\
(0,510)\end{array}$ & $\begin{array}{l}2,602 * * \\
(0,925)\end{array}$ & $\begin{array}{l}0,893 \\
(0,355)\end{array}$ & $\begin{array}{l}1,423 \\
(0,446)\end{array}$ & $\begin{array}{l}1,594 \\
(0,557)\end{array}$ \\
\hline \multicolumn{7}{|c|}{ Gewissenhaftigkeit (Rk.: niedrigstes Quartil) } \\
\hline Zweites Quartil & $\begin{array}{l}1,030 \\
(0,073)\end{array}$ & $\begin{array}{l}0,967 \\
(0,079)\end{array}$ & $\begin{array}{l}0,938 \\
(0,079)\end{array}$ & $\begin{array}{l}0,945 \\
(0,086)\end{array}$ & $\begin{array}{l}0,977 \\
(0,088)\end{array}$ & $\begin{array}{l}1,034 \\
(0,101)\end{array}$ \\
\hline Drittes Quartil & $\begin{array}{l}1,003 \\
(0,066)\end{array}$ & $\begin{array}{l}0,859^{*} \\
(0,066)\end{array}$ & $\begin{array}{l}0,856 \\
(0,069)\end{array}$ & $\begin{array}{l}1,011 \\
(0,077)\end{array}$ & $\begin{array}{l}0,946 \\
(0,078)\end{array}$ & $\begin{array}{l}0,936 \\
(0,081)\end{array}$ \\
\hline Höchstes Quartil & $\begin{array}{l}1,076 \\
(0,103)\end{array}$ & $\begin{array}{l}0,732 * * \\
(0,087)\end{array}$ & $\begin{array}{l}0,681 * * \\
(0,085)\end{array}$ & $\begin{array}{l}0,926 \\
(0,094)\end{array}$ & $\begin{array}{l}0,811 \\
(0,099)\end{array}$ & $\begin{array}{l}0,875 \\
(0,116)\end{array}$ \\
\hline Fehlender Wert & $\begin{array}{l}1,155 \\
(0,319)\end{array}$ & $\begin{array}{l}2,073 * * \\
(0,518)\end{array}$ & $\begin{array}{l}1,794 \\
(0,538)\end{array}$ & $\begin{array}{l}0,938 \\
(0,291)\end{array}$ & $\begin{array}{l}1,718 \\
(0,488)\end{array}$ & $\begin{array}{l}1,832 \\
(0,605)\end{array}$ \\
\hline
\end{tabular}


Tab. 2 (Fortsetzung)

\begin{tabular}{|c|c|c|c|c|c|c|}
\hline \multirow[b]{2}{*}{$\begin{array}{l}\text { Verhältnis der } \\
\text { SuS- zur Eltern- } \\
\text { angabe }\end{array}$} & \multicolumn{3}{|c|}{ Modell 1 (Mütter) } & \multicolumn{3}{|c|}{ Modell 2 (Väter) } \\
\hline & $\begin{array}{l}\text { Inkonsistent/ } \\
\text { konsistent }\end{array}$ & $\begin{array}{l}\text { Keine/ } \\
\text { konsistent }\end{array}$ & $\begin{array}{l}\text { Keine/in- } \\
\text { konsis- } \\
\text { tent }^{\mathrm{a}}\end{array}$ & $\begin{array}{l}\text { Inkonsistent/ } \\
\text { konsistent }\end{array}$ & $\begin{array}{l}\text { Keine/ } \\
\text { konsistent }\end{array}$ & $\begin{array}{l}\text { Keine/in- } \\
\text { konsistent }^{\mathrm{a}}\end{array}$ \\
\hline Fallzahl SuS & 7776 & & & 5859 & & \\
\hline Fallzahl Schulen & 544 & & & 536 & & \\
\hline $\begin{array}{l}\text { LogLikelihood } \\
\text { Startwert }\end{array}$ & -7928 & & & -6198 & & \\
\hline $\begin{array}{l}\text { LogLikelihood } \\
\text { Endwert }\end{array}$ & -7693 & & & -6051 & & \\
\hline $\begin{array}{l}\text { Pseudo- } R^{2} \\
\text { (McFadden) }\end{array}$ & 0,0297 & & & 0,0238 & & \\
\hline
\end{tabular}

Quellen: https://doi.org/10.5157/NEPS:SC4:10.0.0, eigene Berechnungen

Signifikanzniveaus: * $p<0,05 ; * * p<0,01 ; * * * p<0,001$

SuS Schülerinnen und Schüler, $R k$. Referenzkategorie

${ }^{a}$ Bei einer abhängigen Variable mit drei Ausprägungen kann es nur zwei Schätzgleichungen geben. Die Schätzkoeffizienten zum relativen Risiko für keine vs. inkonsistente Angaben ergeben sich durch Division der Schätzkoeffizienten aus den beiden Vorgänger-Spalten. Dies gilt jedoch nicht für die Berechnung der Standardfehler

\subsection{Befunde zu möglichen Einflussfaktoren auf die Übereinstimmung von Eltern und SuS-Angaben in der 9. Jahrgangsstufe}

Auf eine ausführliche Beschreibung der konsistenten, inkonsistenten und fehlenden SuS-Angaben nach den in den multivariaten Modellen berücksichtigten Variablen in den beiden Analysestichproben wird an dieser Stelle verzichtet (detaillierte Übersicht in Tab. 4 im Anhang). Hier konzentrieren wir uns auf die Ergebnisse der multivariaten Schätzung (siehe Tab. 2), insbesondere auf die marginalen Effekte (siehe Abb. 1 und 2).

SuS mit größerem rezeptivem Wortschatz liefern statistisch signifikant häufiger dieselben Angaben zum Bildungsabschluss von Mutter und Vater als SuS mit geringeren Wortschatzkenntnissen. In beiden Stichproben fällt die Differenz für konsistente Angaben bei SuS aus dem höchsten im Vergleich zum niedrigsten Quartil um etwas mehr als 10\%-Punkte höher aus (vgl. Abb. 1 und 2). Im Gegenzug sinkt bei höheren Wortschatzkenntnissen die Wahrscheinlichkeit, dass SuS keine Antwort liefern. Inkonsistenzen in den Angaben sind kaum von Wortschatzkenntnissen abhängig. Nur für SuS aus dem höchsten Quartil werden bei den Angaben zu Vätern (Abb. 2) statistisch signifikant seltener Inkonsistenzen berichtet. Wird nur der Vergleich konsistente vs. inkonsistente Angaben betrachtet (1. und 4. Ergebnisspalte in Tab. 2), so zeigt sich, dass hier sowohl bei Müttern als auch bei Vätern für das oberste Quartil ein auf dem 5\%-Niveau statistisch signifikanter Effekt ausgewiesen wird. Das Risiko einer inkonsistenten im Vergleich zu einer konsistenten Angabe wird um den Faktor 0,765 (bzw. um $(1-0,765) * 100 \%=23,5 \%)$ und um den Faktor 0,667 (bzw. um 33,3\%) reduziert. Die Befunde sind im Sinne von Hypothese 1, wonach höhere Sprachfähigkeit zu einem besseren Verständnis von Fragen und somit zu erhöhten Wahrscheinlichkeiten von Antworten führt. Dass höhere Wortschatz- 


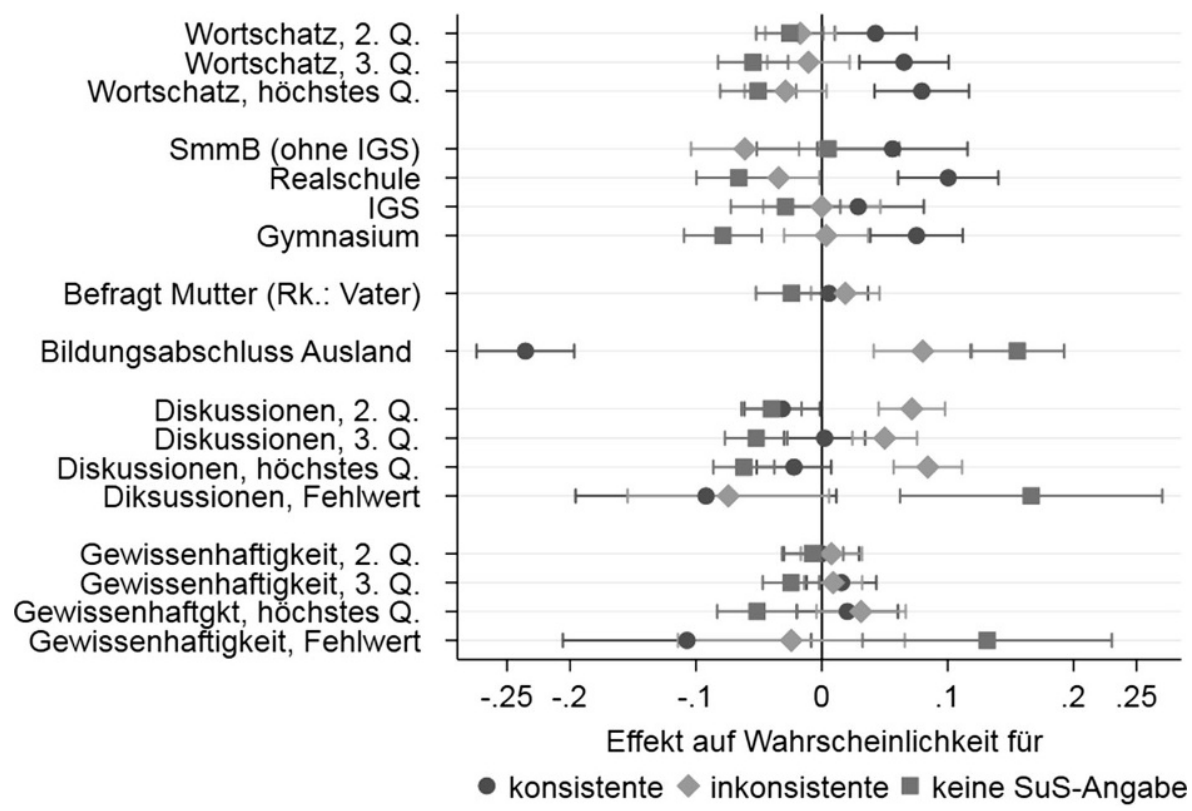

Abb. 1 Differenzen in vorhergesagten Wahrscheinlichkeiten der SuS-Angaben zur Mutter nach ausgewählten Merkmalen (mit 95\%-Konfidenzintervall). Ergebnisse basierend auf Modell 1, Tab. 2. Quellen: https://doi.org/10.5157/NEPS:SC4:10.0.0, eigene Berechnungen. SuS Schülerinnen und Schüler; $R k$. Referenzkategorie; SmmB Schule mit mehreren Bildungsgängen; IGS Integrierte Gesamtschule; Q. Quartil

kenntnisse auch mit „besseren“, d.h. korrekten Antworten einhergehen, lässt sich nur im Vergleich der besonders starken und schwachen SuS zeigen.

Als weiterer Indikator für kognitive Fähigkeiten wurde die Schulformzugehörigkeit verwendet. An Realschulen und Gymnasien werden häufiger konsistente Angaben geliefert als an Hauptschulen, wobei SuS an Gymnasien nicht besser abschneiden als Realschüler. Gleichzeitig sind die SuS an diesen beiden Schularten auch die, die am seltensten keine Angabe liefern. Zugleich haben SuS an Realschulen und Gymnasien ähnlich häufig inkonsistente Angaben wie SuS an Hauptschulen (vgl. Abb. 1 und 2). Das Antwortverhalten an Integrierten Gesamtschulen scheint, unter Kontrolle der weiteren Merkmale, vergleichbar mit dem an Hauptschulen zu sein.

Nach Hypothese 2 sollte die Übereinstimmung höher sein, wenn das Elternteil selbst befragt wurde. Diese Hypothese bezieht sich ausschließlich auf den Vergleich inkonsistenter vs. konsistenter Angaben. Die Wahrscheinlichkeit, dass SuS überhaupt eine Antwort liefern, sollte davon unberührt sein. Wie anhand von Abb. 1 und $2 \mathrm{zu}$ erkennen, sind die Unterschiede in den Antworten sehr gering und zufallskritisch nicht abgesichert (siehe auch Tab. 2). Die Befunde widersprechen klar Hypothese 2.

Haben Eltern ihren Bildungsabschluss im Ausland erworben, steigt die Wahrscheinlichkeit um fast 20\%-Punkte, dass ihr Kind keine Angabe in der schriftlichen Befragung liefert. Im Fall von Müttern steigt auch die Wahrscheinlichkeit statistisch signifikant, inkonsistente Angaben zu liefern, bei Vätern umschließt das $95 \%$ - 


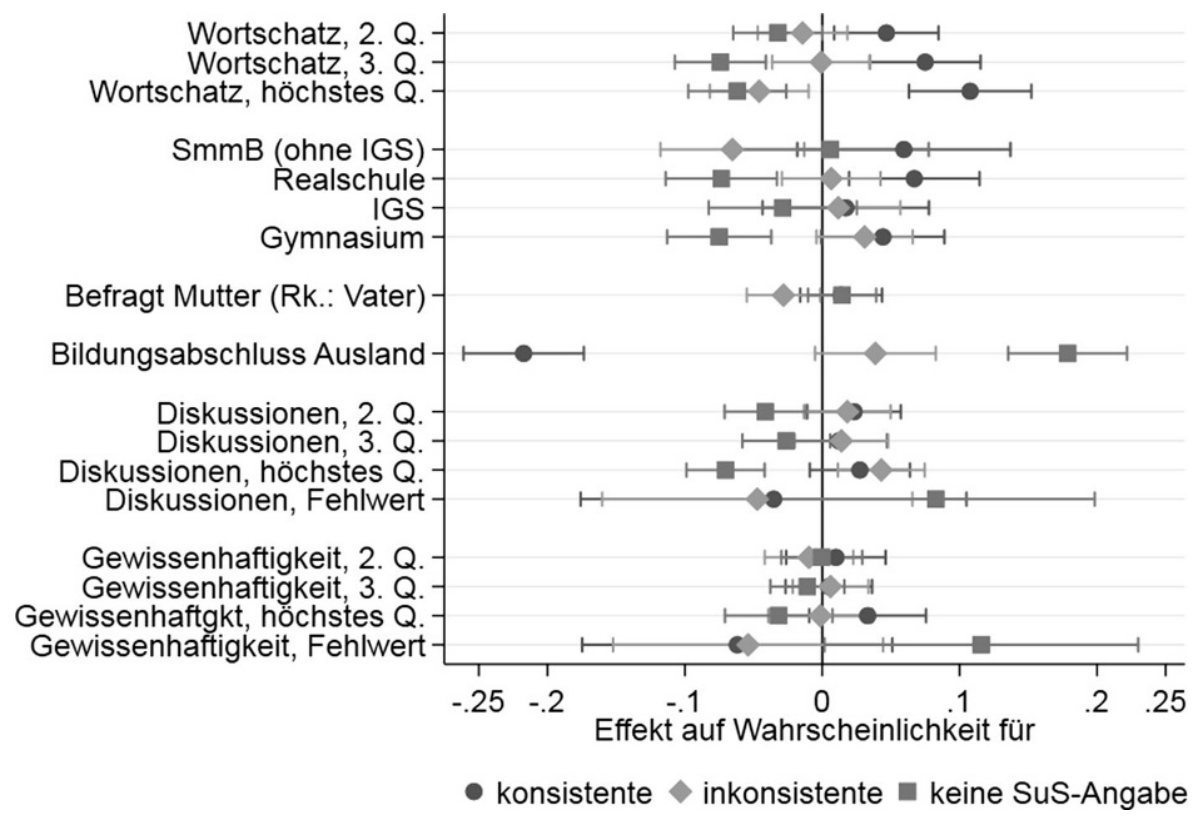

Abb. 2 Differenzen in vorhergesagten Wahrscheinlichkeiten der SuS-Angaben zum Vater nach ausgewählten Merkmalen (mit 95\%-Konfidenzintervall). Ergebnisse basierend auf Modell 2, Tab. 2. Quellen: https://doi.org/10.5157/NEPS:SC4:10.0.0, eigene Berechnungen. SuS Schülerinnen und Schüler; $R k$. Referenzkategorie; SmmB Schule mit mehreren Bildungsgängen; IGS Integrierte Gesamtschule; Q. Quartil

Konfidenzinterfall die Null. Dagegen sinkt in beiden Fällen die Wahrscheinlichkeit konsistenter Angaben erheblich (Abb. 1 und 2). Jugendlichen fällt es offenbar besonders schwer, Bildungsangaben zu den Eltern zu liefern, wenn diese im Ausland erworben wurden. H3 kann somit nicht falsifiziert werden.

Nach Hypothese 4 sollten SuS, die mehr mit ihren Eltern kommunizieren, ein stärker verankertes Wissen über deren Bildungsabschlüsse besitzen und dieses leichter abrufen können. Entgegen dieser Erwartung zeigen Abb. 1 und 2, dass mehr Diskussionen im Elternhaus zwar mit geringeren Wahrscheinlichkeiten einhergehen, keine Angabe zu liefern, nicht aber zu insgesamt besseren Übereinstimmungen führen. Die Annahme, dass ein reger Austausch in den Familien auch automatisch zu einer kognitiv besseren Verankerung der Lebens- und Bildungswege der Eltern führt, lässt sich nicht bestätigen. Hypothese 4 muss also verworfen werden.

Personen, die besonders gewissenhaft sind, liefern häufiger Angaben zur Bildung der Eltern. Offenbar sind sie motivierter, kognitive Ressourcen aufzuwenden, um eine Antwort zu finden. Dies führt aber nicht dazu, dass die Übereinstimmung in den Angaben steigt. Deshalb muss die fünfte Hypothese zum Teil verworfen werden. 
Tab. 3 Lineare Regression der Mathematikkompetenzen von SuS auf den Bildungsabschluss der Mutter und des Vaters (jeweils identische Stichprobe), robuste Standardfehler in Klammern

\begin{tabular}{|c|c|c|c|c|c|c|}
\hline $\begin{array}{l}\text { Auskunft gebende } \\
\text { Person: }\end{array}$ & $\begin{array}{l}\text { Modell } 3 \\
\text { (Mütter) } \\
\text { Elter }\end{array}$ & $\begin{array}{l}\text { Modell } 4 \\
\text { (Mütter) } \\
\text { SuS }\end{array}$ & $\begin{array}{l}\text { Differenz } \\
\text { M3-M4 }\end{array}$ & $\begin{array}{l}\text { Modell } 5 \\
\text { (Väter) } \\
\text { Elter }\end{array}$ & $\begin{array}{l}\text { Modell } 6 \\
\text { (Väter) } \\
\text { SuS }\end{array}$ & $\begin{array}{l}\text { Differenz } \\
\text { M5-M6 }\end{array}$ \\
\hline \multicolumn{7}{|c|}{ Höchster Bildungsabschluss (Rk.: Hauptschulabschluss) } \\
\hline Kein Abschluss & $\begin{array}{l}-0,477 * * * \\
(0,081)\end{array}$ & $\begin{array}{l}-0,412 * * * \\
(0,091)\end{array}$ & $\begin{array}{l}-0,065 \\
(0,101)\end{array}$ & $\begin{array}{l}-0,638 * * * \\
(0,175)\end{array}$ & $\begin{array}{l}-0,561 * * * \\
(0,107)\end{array}$ & $\begin{array}{l}-0,078 \\
(0,166)\end{array}$ \\
\hline Mittlere Reife & $\begin{array}{l}0,360 * * * \\
(0,036)\end{array}$ & $\begin{array}{l}0,147 * * * \\
(0,036)\end{array}$ & $\begin{array}{l}0,213 * * * \\
(0,031)\end{array}$ & $\begin{array}{l}0,197 * * * \\
(0,041)\end{array}$ & $\begin{array}{l}0,029 \\
(0,038)\end{array}$ & $\begin{array}{l}0,168 * * * \\
(0,037)\end{array}$ \\
\hline (Fach-)Abitur & $\begin{array}{l}0,715 * * * \\
(0,046)\end{array}$ & $\begin{array}{l}0,575 * * * \\
(0,046)\end{array}$ & $\begin{array}{l}0,140 * * * \\
(0,035)\end{array}$ & $\begin{array}{l}0,523 * * * \\
(0,053)\end{array}$ & $\begin{array}{l}0,413 * * * \\
(0,048)\end{array}$ & $\begin{array}{l}0,110 * \\
(0,043)\end{array}$ \\
\hline $\begin{array}{l}\text { (Fach-)Hochschulab- } \\
\text { schluss }\end{array}$ & $\begin{array}{l}0,947 * * * \\
(0,052)\end{array}$ & $\begin{array}{l}0,695 * * * \\
(0,053)\end{array}$ & $\begin{array}{l}0,252 * * * \\
(0,039)\end{array}$ & $\begin{array}{l}0,833 * * * \\
(0,047)\end{array}$ & $\begin{array}{l}0,701 * * * \\
(0,051)\end{array}$ & $\begin{array}{l}0,133 * * * \\
(0,036)\end{array}$ \\
\hline Konstante & $\begin{array}{l}-0,462 * * * \\
(0,036)\end{array}$ & $\begin{array}{l}-0,299 * * * \\
(0,040)\end{array}$ & - & $\begin{array}{l}-0,361 * * * \\
(0,037)\end{array}$ & $\begin{array}{l}-0,239 * * * \\
(0,039)\end{array}$ & - \\
\hline Fallzahl SuS & 6137 & 6137 & & 4393 & 4393 & \\
\hline Fallzahl Schulen & 536 & 536 & & 519 & 519 & \\
\hline$R^{2}$ & 0,1050 & 0,0734 & & 0,1140 & 0,0872 & \\
\hline
\end{tabular}

Quellen: https://doi.org/10.5157/NEPS:SC4:10.0.0, eigene Berechnungen

Signifikanzniveaus: $* p<0,05 ; * * p<0,01 ; * * * p<0,001$

SuS Schülerinnen und Schüler, $R k$. Referenzkategorie

\subsection{Befunde zur Über- oder Unterschätzung von Bildungsungleichheiten bei Verwendung von SuS- anstelle von Elternangaben}

Modell 3 und 4, Tab. 3, geben die Ergebnisse zum Zusammenhang von Bildungsabschluss der Mutter und den Mathematikkompetenzen wieder. Die Regressionskoeffizienten fallen - wie zu erwarten - in aufsteigender Folge aus. Je höher der Bildungsabschluss der Mutter ausfällt, desto größere Mathematikkompetenzen sind bei ihren Kindern zu beobachten. Während sich der Effekt für die unterste Bildungsgruppe (kein Bildungsabschluss vs. Hauptschulabschluss) zwischen den beiden Modellen kaum unterscheidet, sind die Differenzen bei den anderen drei Bildungsgruppen deutlich größer. In dem Modell mit den Bildungsangaben, die von den Eltern geliefert werden, fällt der Unterschied zwischen Akademikerinnen und Hauptschulabsolventinnen um 0,25 Standardabweichungen, bei Müttern mit Realschul- im Vergleich zu Hauptschulabschluss um 0,21 Standardabweichungen größer aus als in dem Modell mit SuS-Angaben. Diese Differenzen sind auf dem 0,1\%-Niveau statistisch signifikant (vgl. Tab. 3, Spalte 4). Für diese beiden Bildungsgruppen wird das Ausmaß der Ungleichheit bei Verwendung von SuS-Angaben also massiv unterschätzt.

Werden die Zusammenhänge für die Angaben zu den Vätern betrachtet, zeigt sich ein vergleichbares Muster. Auch hier fallen die Ungleichheiten bei Verwendung der SuS-Angaben geringer aus. Die Unterschiede sind aber mit 0,13 bzw. 0,17 
Standardabweichung für Akademiker bzw. Realschul- vs. Hauptschulabsolventen etwas schwächer (siehe Tab. 3, letzte Spalte).

Im Anhang präsentieren wir noch die Ergebnisse für den Fall, dass das bisher verwendete Kriterium für den Stichprobenzuschnitt ,gemeinsames Vorliegen von SuS- und Eltern-Angabe“ aufgegeben wird. Dann betragen die Fallzahlen in den Analysestichproben zu Müttern 7926 bei Eltern- und 10.776 bei SuS-Angaben, in der zu Vätern 6176 und 9899 Fälle. Beibehalten wird jedoch die zuvor gewählte Normierung der Mathematikleistungen. Die in Tab. 7 präsentierten Befunde aus den sich nur teilweise überlappenden Analysestichproben sind vergleichbar zu denen in Tab. 3. ${ }^{6}$ Tendenziell sind die Unterschiede für Akademikermütter und -väter bei Verwendung der ,größtmöglichen“ Fallzahlen sogar etwas größer als bei Verwendung eines gemeinsamen Stichprobenzuschnitts.

\section{Diskussion}

Die Untersuchungen konnten die Ergebnisse von Kreuter et al. (2006, 2010) sowie Jerrim und Micklewright (2014) replizieren und erweitern. 15-Jährige in Regelschulen sind offenbar nur im begrenzten Umfang in der Lage, Bildungsabschlüsse ihrer Eltern in standardisierten Befragungen korrekt zu berichten. In den hier verwendeten Analysestichproben gelingt dies in etwa nur jedem zweiten SuS.

Unter Heranziehung der kognitiven Theorie des Antwortverhaltens wurden die vier Phasen der Beantwortung von Fragen in standardisierten Erhebungen diskutiert und Hypothesen hergeleitet. Diese bezogen sich größtenteils (H1 bis H4) auf das Vermögen, die Frage korrekt zu beantworten. Je höher das Vermögen, desto geringer die erforderlichen kognitiven Ressourcen für eine korrekte Beantwortung der Frage. Die Motivation, kognitive Ressourcen zu aktivieren, wurde durch Hypothese (H5) abgedeckt. In der ersten Phase muss die Frage zunächst verstanden werden. Werden Teile der Frage (Fragetext, Ausfüllhinweise, Antwortkategorien) nicht verstanden, könnte dies zu höherem Item-Nonresponse oder zum willkürlichen Setzen von Kreuzen (unachtsames Antworten) führen. Die Befunde, wonach höhere Wortschatzkenntnisse die Wahrscheinlichkeit steigern, überhaupt und zudem häufiger konsistent zu antworten, passen hierzu. Den beiden nächsten Phasen, Suche und Bewertung von Informationen, haben wir uns zum einen über die Verankerung von Informationen und Anstrengungen in der Informationssuche genähert. Hier wurden zum einen im Ausland erworbene Bildungsabschlüsse und Diskussionen mit den Eltern sowie die Persönlichkeitseigenschaft Gewissenhaftigkeit als Indikatoren verwendet. Haben die Eltern ihren Bildungsabschluss im Ausland erworben, fällt es vielen $\mathrm{SuS}$ schwer, überhaupt eine Antwort zu geben. Entweder ist ihnen die Information nicht präsent oder ihnen gelingt es nicht, die Information in die Ant-

\footnotetext{
${ }^{6}$ Zusätzlich wurden die Modelle noch mithilfe der NEPS-Gewichte für SuS und für gemeinsame Teilnahme von SuS und Eltern geschätzt (zu den Gewichten siehe auch Fußnote 4). Die gewichteten Schätzergebnisse unterscheiden sich nur unwesentlich von den in Tab. 7 präsentierten Ergebnissen (Ergebnisse auf Anfrage erhältlich). Einschränkend ist darauf hinzuweisen, dass bei Verwendung von Gewichten, die über Modelle variieren, keine Standardfehler mithilfe von SUEST (siehe Ende von Kap. 3) geschätzt werden können.
} 
wortkategorien, die sich auf deutsche Abschlüsse beziehen, einzuordnen. Häufige Diskussionen mit den Eltern zu künstlerischen, sozialen und politischen Themen reduzieren zwar den Item-Nonresponse, führen aber nicht zu korrekteren Angaben. Dass sich Personen, die sich durch hohe Gewissenhaftigkeit auszeichnen, besonders viel Mühe bei der Beantwortung von Fragen geben und z. B. vor Satisficing, dem Begnügen mit der ,erst besten“ Antwort, gefeit sind, zeigt sich nur ansatzweise. Besonders gewissenhafte SuS verweigern in der Tat etwas seltener Antworten zu ihrer Mutter, sie scheinen motivierter, kognitive Ressourcen aufzuwenden. Bei der Angabe zum Vater spielt Gewissenhaftigkeit keine Rolle. Die Konsistenz der Antworten variiert aber in beiden Stichproben nicht mit der Gewissenhaftigkeit von SuS.

Elternteile müssten der kognitiven Theorie des Antwortverhaltens zufolge die eigenen Bildungsabschlüsse besser erinnern als die des Partners/der Partnerin. Deshalb sollte mehr „Rauschen“ in den Angaben zu dem Elternteil, über das berichtet wurde, vorliegen und folglich geringere Übereinstimmungen mit den SuS. Die Analysen konnten dies nicht bestätigen. Selbstverständlich lässt sich hieraus nicht ableiten, dass Selbsterlebtes, das möglicherweise von zentraler Bedeutung für die Identitätsbildung war und ist, generell nicht besser abgerufen werden kann als biographische Ereignisse und Begebenheiten einer Partnerin oder eines Partners. Vielleicht liegt es an der großen Bedeutung von formalen Bildungszertifikaten für die Positionierung auf dem deutschen Arbeitsmarkt sowie möglicherweise auch an (Aus-)Bildungsstätten als Ort der Partnerfindung (Blossfeld und Timm 2003), dass Eltern so gute Kenntnisse zum Bildungsabschluss des anderen Elternteils haben. Die letzte Phase der kognitiven Theorie des Antwortverhaltens konnten wir nicht in den Blick nehmen.

Das Erhebungsprogramm des von uns verwendeten Datensatzes ist nicht speziell auf die Prüfung der kognitiven Theorie des Antwortverhaltens ausgelegt. So ist es nicht möglich, die verschiedenen Phasen einzeln zu betrachten. Erhebungen, die z.B. Probing, Antwortlatenzen, Eye-tracking, offene und geschlossenen Antwortformate im Vergleich oder alternative Frageformulierungen enthalten, sollten dafür besser geeignet sein. Auch fehlen die in der Literatur vorgeschlagenen standardisierten Fragen zur Identifizierung unachtsamen Antwortens oder Selbstauskünfte zu den aufgewendeten Anstrengungen beim Bearbeiten der Fragen (Meade und Craig 2012, S. 452). Zudem ist die Operationalisierung einiger Konstrukte wie die Verankerung von Informationen über Diskussionen mit den Eltern nicht optimal, und die Reliabilität der Skala zu Gewissenhaftigkeit ist niedrig. Dennoch liefert die kognitive Theorie des Antwortverhaltens einen sinnvollen Rahmen, um SuS-Angaben zu Bildungsabschlüssen der Eltern zu untersuchen. Darüber hinaus lässt sich aus den Analysen schlussfolgern, dass SuS, die korrekte Angaben liefern, in mehrerlei Hinsicht selektiv sind. Sie verfügen z. B. über höhere Sprachkenntnisse und ihre Eltern haben das Bildungssystem häufiger in Deutschland durchlaufen. Folglich ist davon auszugehen, dass die Nutzung der SuS-Angaben zum Bildungsstand der Eltern bei verschiedensten Fragestellungen zu verzerrten Schätzergebnissen führt. Dass die Übereinstimmungsraten von SuS und Eltern im Falle von Proxy-Angaben der Eltern zum zweiten Elter genauso hoch sind, wie bei eigenen Angaben der Eltern deutet darauf hin, dass hier Proxy-Angaben der Eltern valide sind. Es kann jedoch 
nicht ausgeschlossen werden, dass befragte Eltern und ihre Kinder den gleichen systematischen Fehler begehen.

Die vergleichenden Auswertungen zu sozialen Ungleichheiten in Mathematikkompetenzen zeigen, dass bei Verwendung von SuS-Angaben Ungleichheiten deutlich unterschätzt werden. Von der Verwendung dieser SuS-Angaben ist deshalb abzuraten. Welche praktischen Konsequenzen ergeben sich daraus? Erstens, Analysen mit den SuS-Daten sollten auf Elternangaben zurückgreifen, falls Informationen zum Bildungsabschluss in den Analysen verwendet werden. Im Fall von NEPS SC4 enthalten die Elterndaten zudem differenziertere Angaben zur beruflichen Bildung. Nun haben bei NEPS SC4, wie auch in anderen Befragungen, nicht alle Eltern an der Befragung teilgenommen. Zudem gibt es auch auf Seiten der SuS Item-Nonresponse. Von den 14.490 SuS in Regelschulen, die in der ersten Welle teilgenommen haben, lieferten 10.776 Angaben zum Bildungsabschluss der Mutter. Von Seiten der Eltern liegen aber nur Angaben zu 7926 Müttern vor. Bei Vätern betragen die Fallzahlen 9899 und 6176 (für Anzahl SuS in Regelschulen siehe Tab. 1, für die anderen Zahlen Tab. 7 im Anhang). Bei Verwendung der Elternangabe droht also ein erheblicher und zudem selektiver Verlust an Fällen. Eine Option, dies zu vermeiden, sind multiple Imputationen. Hier könnten auch die SuS-Angaben zu den Bildungsabschlüssen der Eltern neben weiteren Informationen wie Ausstattung des Haushaltes mit Büchern oder berufliche Tätigkeit der Eltern, die ebenfalls im SuSFragebogen erhoben werden, als aussagekräftige Prädiktoren zur Vorhersage der Bildung der Eltern im Imputationsmodell berücksichtigt werden.

Eine zweite praktische Konsequenz ist, dass in Schulerhebungen auf Elternbefragungen nicht verzichtet werden sollte, auch wenn dies mit höheren Kosten einhergeht - darin schließen wir uns Kreuter et al. an, die vorschlagen: ,whenever possible, parent reports should be used“" (2010, S. 135). Hieraus ergibt sich die Frage, ob diese Forderung auch für Studierendenbefragungen oder andere Befragungen mit jungen Erwachsenen gelten sollte bzw. ab welchem Alter damit zu rechnen ist, dass Befragte valide(re) Informationen zu den Bildungsabschlüssen der Eltern liefern können. Daten mit geringer Qualität haben Folgen. Selbst bei einer einfachen Beschreibung von Zusammenhängen wie dem Ausmaß von Bildungsungleichheit, führen Messfehler i.d.R. zu einer Untererfassung der Ungleichheit. Als letztes empfehlen wir, in Panelstudien die Bildung der Eltern nicht nur einmalig, sondern wiederholt zu erheben, damit die Anzahl an Fällen ohne Informationen (Item-Nonresponse) reduziert wird.

\section{Anhang}




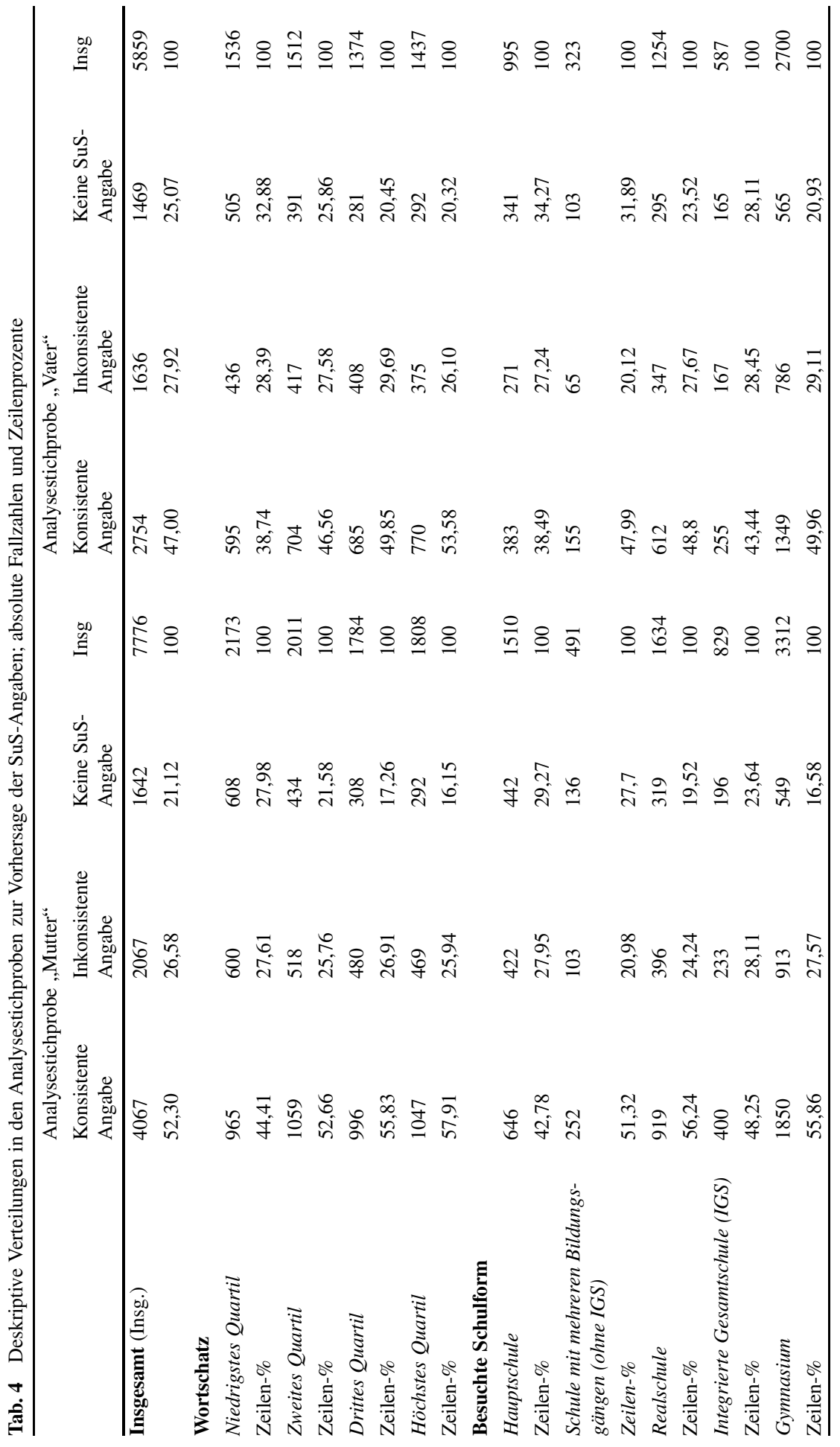




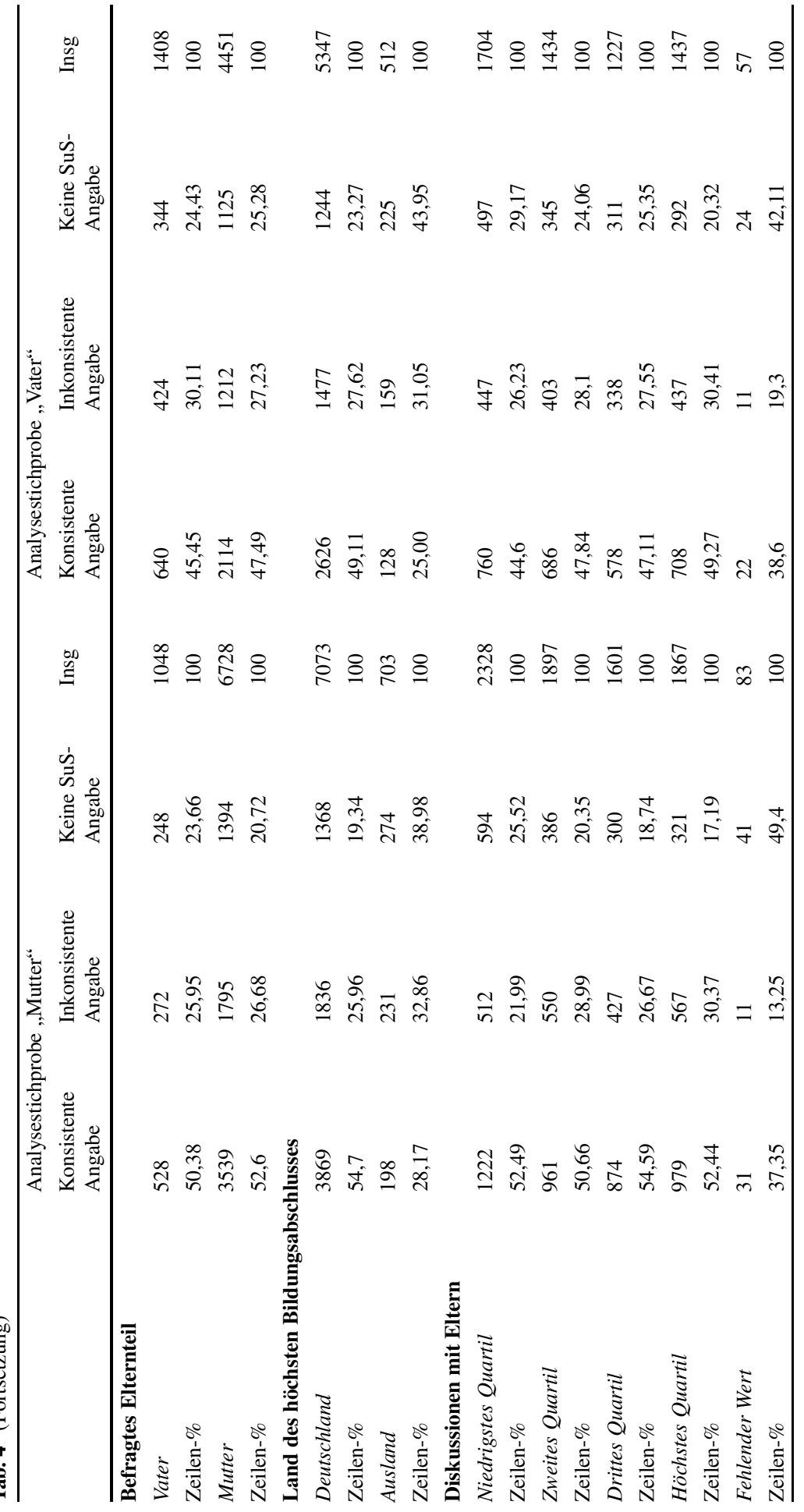




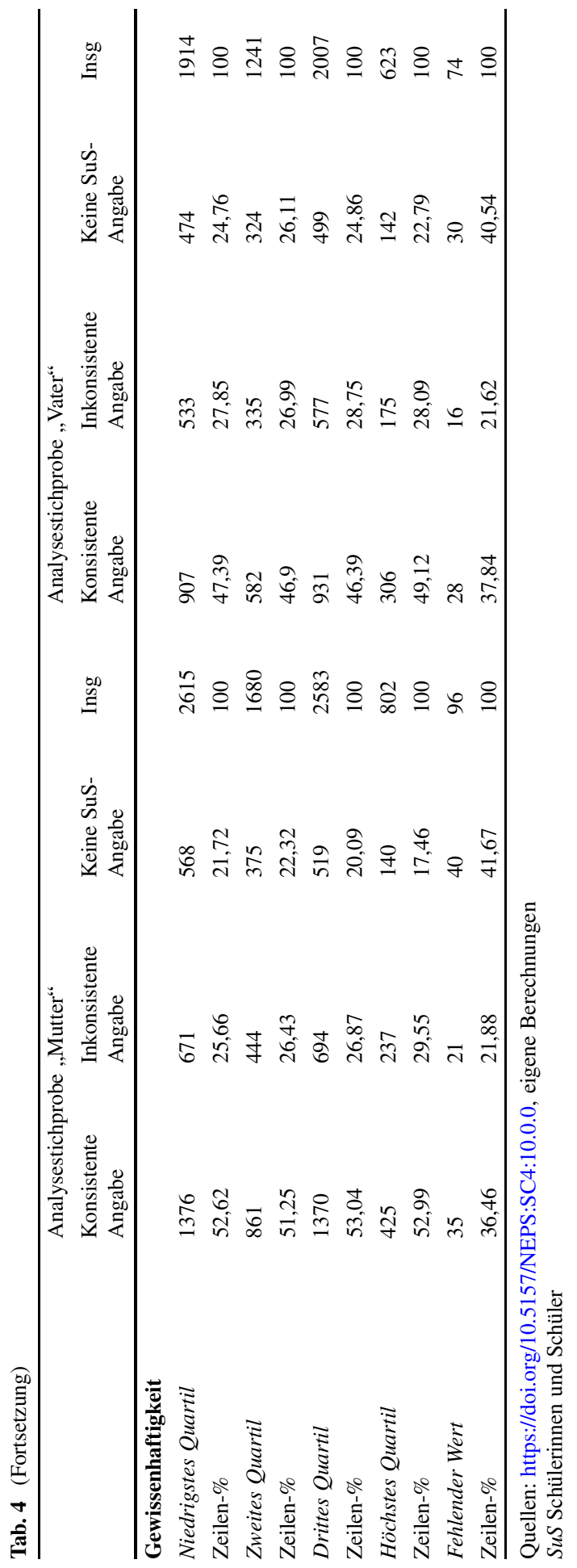


Tab. 5 Prozentuale Übereinstimmungen (PÜ) und Cohens Kappa zur Bildung der Eltern, Standardfehler in Klammern

\begin{tabular}{lll}
\hline & Bildung Mutter & Bildung Vater \\
\hline PÜ (in Prozent) & $66,3 \%$ & $62,7 \%$ \\
PÜ (relativ) & 0,663 & 0,627 \\
& $(0,006)$ & $(0,007)$ \\
Cohens Kappa & 0,522 & 0,503 \\
& $(0,008)$ & $(0,010)$ \\
Fallzahl & 6134 & 4390 \\
\hline
\end{tabular}

Quellen: https://doi.org/10.5157/NEPS:SC4:10.0.0, eigene Berechnungen

Tab. 6 Verteilung der Bildungsabschlüsse in den verschiedenen Stichproben zur Vorhersage der Mathematikkompetenzen

\begin{tabular}{|c|c|c|c|c|c|c|c|c|}
\hline $\begin{array}{l}\text { Auskunft ge- } \\
\text { bende Person: }\end{array}$ & $\begin{array}{l}\text { Modell } 3 \\
\text { (Mütter) } \\
\text { Elter }\end{array}$ & $\begin{array}{l}\text { Modell } 4 \\
\text { (Mütter) } \\
\text { SuS }\end{array}$ & $\begin{array}{l}\text { Modell } 5 \\
\text { (Väter) } \\
\text { Elter }\end{array}$ & $\begin{array}{l}\text { Modell } 6 \\
\text { (Väter) } \\
\text { SuS }\end{array}$ & $\begin{array}{l}\text { Modell } 7 \\
\text { (Mütter) } \\
\text { Elter }\end{array}$ & $\begin{array}{l}\text { Modell } 8 \\
\text { (Mütter) } \\
\text { SuS }\end{array}$ & $\begin{array}{l}\text { Modell } 9 \\
\text { (Väter) } \\
\text { Elter }\end{array}$ & $\begin{array}{l}\text { Modell } 10 \\
\text { (Väter) } \\
\text { SuS }\end{array}$ \\
\hline \multicolumn{9}{|c|}{ Höchster Bildungsabschluss } \\
\hline $\begin{array}{l}\text { Kein Ab- } \\
\text { schluss }\end{array}$ & 56 & 100 & 21 & 60 & 94 & 381 & 46 & 286 \\
\hline $\begin{array}{l}\text { Hauptschul- } \\
\text { abschluss }\end{array}$ & 1108 & 915 & 1212 & 962 & 1574 & 1930 & 1763 & 2402 \\
\hline Mittlere Reife & 2648 & 2751 & 1304 & 1485 & 3367 & 4879 & 1855 & 3572 \\
\hline (Fach-)Abitur & 1272 & 1456 & 652 & 974 & 1567 & 2226 & 901 & 1956 \\
\hline $\begin{array}{l}\text { (Fach-)Hoch- } \\
\text { schulabschluss }\end{array}$ & 1053 & 915 & 1204 & 912 & 1324 & 1360 & 1611 & 1683 \\
\hline Fallzahl SuS & 6137 & 6137 & 4393 & 4393 & 7926 & 10.776 & 6176 & 9899 \\
\hline Fallzahl Schulen & 536 & 536 & 519 & 519 & 544 & 544 & 537 & 544 \\
\hline
\end{tabular}

Quellen: https://doi.org/10.5157/NEPS:SC4:10.0.0, eigene Berechnungen 
Tab. 7 Lineare Regression der Mathematikkompetenzen von SuS auf den Bildungsabschluss von Mutter und Vaters (jeweils alle Fälle mit Angaben zur elterlichen Bildung)

\begin{tabular}{|c|c|c|c|c|c|c|}
\hline $\begin{array}{l}\text { Auskunftgebende } \\
\text { Person }\end{array}$ & $\begin{array}{l}\text { Modell } 7 \\
\text { (Mütter) } \\
\text { Elter }\end{array}$ & Modell 8 & $\begin{array}{l}\text { Differenz } \\
\text { M7-M8 }\end{array}$ & $\begin{array}{l}\text { Modell } 9 \\
\text { (Väter) } \\
\text { Elter }\end{array}$ & Modell 10 & $\begin{array}{l}\text { Differenz } \\
\text { M9-M10 }\end{array}$ \\
\hline \multicolumn{7}{|c|}{ Höchster Bildungsabschluss (Rk.: Hauptschulabschluss) } \\
\hline Kein Abschluss & $\begin{array}{l}-0,404 * * * \\
(0,068)\end{array}$ & $\begin{array}{l}-0,417 * * * \\
(0,048)\end{array}$ & $\begin{array}{l}0,013 \\
(0,071)\end{array}$ & $\begin{array}{l}-0,665^{* * *} \\
(0,102)\end{array}$ & $\begin{array}{l}-0,477 \text { *** } \\
(0,051)\end{array}$ & $\begin{array}{l}-0,188 \\
(0,107)\end{array}$ \\
\hline Mittlere Reife & $\begin{array}{l}0,387 * * * \\
(0,032)\end{array}$ & $\begin{array}{l}0,178 * * * \\
(0,027)\end{array}$ & $\begin{array}{l}0,209 * * * \\
(0,029)\end{array}$ & $\begin{array}{l}0,207 * * * \\
(0,034)\end{array}$ & $\begin{array}{l}0,026 \\
(0,026)\end{array}$ & $\begin{array}{l}0,180 * * * \\
(0,035)\end{array}$ \\
\hline (Fach-)Abitur & $\begin{array}{l}0,739 * * * \\
(0,042)\end{array}$ & $\begin{array}{l}0,570^{* * * *} \\
(0,039)\end{array}$ & $\begin{array}{l}0,169 * * * \\
(0,035)\end{array}$ & $\begin{array}{l}0,521 * * * \\
(0,045)\end{array}$ & $\begin{array}{l}0,395 * * * \\
(0,035)\end{array}$ & $\begin{array}{l}0,126^{* *} \\
(0,042)\end{array}$ \\
\hline $\begin{array}{l}\text { (Fach-)Hochschul- } \\
\text { abschluss }\end{array}$ & $\begin{array}{l}0,998 * * * \\
(0,049)\end{array}$ & $\begin{array}{l}0,703 * * * \\
(0,046)\end{array}$ & $\begin{array}{l}0,295 * * * \\
(0,041)\end{array}$ & $\begin{array}{l}0,833 * * * \\
(0,042)\end{array}$ & $\begin{array}{l}0,618 * * * \\
(0,040)\end{array}$ & $\begin{array}{l}0,215^{* * * *} \\
(0,036)\end{array}$ \\
\hline Konstante & $\begin{array}{l}-0,528 * * * \\
(0,031)\end{array}$ & $\begin{array}{l}-0,418 * * * \\
(0,033)\end{array}$ & - & $\begin{array}{l}-0,423 * * * \\
(0,033)\end{array}$ & $\begin{array}{l}-0,380 * * * \\
(0,032)\end{array}$ & - \\
\hline Fallzahl SuS & 7926 & 10.776 & & 6176 & 9899 & \\
\hline Fallzahl Schulen & 544 & 544 & & 537 & 544 & \\
\hline$R^{2}$ & 0,1160 & 0,0785 & & 0,1150 & 0,0756 & \\
\hline
\end{tabular}

Quellen: https://doi.org/10.5157/NEPS:SC4:10.0.0, eigene Berechnungen

Signifikanzniveaus: $* p<0,05 ; * * p<0,01 ; * * * p<0,001$

$R k$. Referenzkategorie

Funding Open Access funding enabled and organized by Projekt DEAL.

Open Access Dieser Artikel wird unter der Creative Commons Namensnennung 4.0 International Lizenz veröffentlicht, welche die Nutzung, Vervielfältigung, Bearbeitung, Verbreitung und Wiedergabe in jeglichem Medium und Format erlaubt, sofern Sie den/die ursprünglichen Autor(en) und die Quelle ordnungsgemäß nennen, einen Link zur Creative Commons Lizenz beifügen und angeben, ob Änderungen vorgenommen wurden.

Die in diesem Artikel enthaltenen Bilder und sonstiges Drittmaterial unterliegen ebenfalls der genannten Creative Commons Lizenz, sofern sich aus der Abbildungslegende nichts anderes ergibt. Sofern das betreffende Material nicht unter der genannten Creative Commons Lizenz steht und die betreffende Handlung nicht nach gesetzlichen Vorschriften erlaubt ist, ist für die oben aufgeführten Weiterverwendungen des Materials die Einwilligung des jeweiligen Rechteinhabers einzuholen.

Weitere Details zur Lizenz entnehmen Sie bitte der Lizenzinformation auf http://creativecommons.org/ licenses/by/4.0/deed.de.

\section{Literatur}

Autorengruppe Bildungsberichterstattung (2020). Bildung in Deutschland 2020. Ein indikatorengestützter Bericht mit einer Analyse zu Bildung in einer digitalisierten Welt. Bielefeld: wbv.

Barrick, M.R., \& Mount, M. K. (1991). The big five personality dimensions and job performance: a metaanalysis. Personnel psychology, 44, 1-26.

Blossfeld, H.-P., \& Roßbach, H.-G. (Hrsg.). (2019). Education as a Lifelong Process-The German National Educational Panel Study (NEPS). Wiesbaden: Springer VS. 
Blossfeld, H.-P., \& Timm, A. (2003). Who marries whom? Educational systems as marriage markets in modern societies. Dordrecht: Springer.

Bradbury, B., Corak, M., Waldfogel, J., \& Washbrook, E. (2015). Too many children left behind: the US achievement gap in comparative perspective. New York: SAGE.

Clogg, C. C., Petkova, E., \& Haritou, A. (1995). Statistical methods for comparing regression coefficients between models. American Journal of Sociology, 100, 1261-1293.

Curran, P.G. (2016). Methods for the detection of carelessly invalid responses in survey data. Journal of Experimental Social Psychology, 66, 4-19.

Duchhardt, C., \& Gerdes, A. (2013). NEPS technical report for mathematics—scaling results of starting cohort 4 in ninth grade (NEPS Working Paper No., Bd. 22). Bamberg: University of Bamberg, National Educational Panel Study.

Dunn, L. M., \& Dunn, L. M. (2004). Peabody Picture Vocabulary Test (PPVT) (deutsche Version). Göttingen: Hogrefe.

Engzell, P., \& Jonsson, J.O. (2015). Estimating social and ethnic inequality in school surveys: biases from child misreporting and parent nonresponse. European Sociological Review, 31, 312-325.

Ensminger, M.E., Forrest, C. B., Riley, A. W., Kang, M., Green, B. F., Starfield, B., \& Ryan, S. A. (2000). The validity of measures of socioeconomic status of adolescents. Journal of Adolescent Research, 15, $392-419$.

Erikson, R. (2019). How does education depend on social origin? In R. Becker (Hrsg.), Research handbook on the sociology of education (S. 35-56). Cheltenham: Edward Elgar.

De Feyter, T., Caers, R., Vigna, C., \& Berings, D. (2012). Unraveling the impact of the Big Five personality traits on academic performance: The moderating and mediating effects of self-efficacy and academic motivation. Learning and individual Differences, 22, 439-448.

Flohr, M., Menze, L., \& Protsch, P. (2020). Berufliche Aspirationen im Kontext regionaler Berufsstrukturen. Kölner Zeitschrift für Soziologie und Sozialpsychologie, 72, 79-104.

Guill, K., Lüdtke, O., \& Köller, O. (2020). Assessing the instructional quality of private tutoring and effects on student outcomes: analyses from the German National Educational Panel Study. British Journal of Educational Psychology, 90(2), 282-300.

Hooper, M., Mullis, I., Mullin, M., \& Fishbein, B. (2017). TIMSS 2019 context questionnaire framework. In I. Mullis \& M. Martin (Hrsg.), TIMSS 2019 assessment frameworks (S. 57-78). Boston: IEA.

Hußmann, A., Wendt, H., Kasper, D., Bos, W., \& Goy, M. (2017). IGLU 2016: Ziele, Anlage und Durchführung der Internationalen Grundschul-Lese-Untersuchung (IGLU 2016). In A. Hußmann, H. Wendt, W. Bos, A. Bremerich-Vos, D. Kasper \& E.-M. Lankes (Hrsg.), IGLU 2016: Lesekompetenzen von Grundschulkindern in Deutschland im internationalen Vergleich (S. 29-78). Münster: Waxmann.

IEA DPC Methodenbericht - NEPS Startkohorte 4 - Förderschulen. Haupterhebung - Herbst/Winter 2010. Hamburg: IEA DPC. https://www.neps-data.de/Portals/0/NEPS/Datenzentrum/Forschungsdaten/ SC4/Methodenbericht_A60,A86.pdf. Zugegriffen: 20. Jan. 2020.

IEA DPC Methodenbericht - NEPS Startkohorte 4. Haupterhebung - Herbst/Winter 2010. Hamburg: IEA DPC. https://www.neps-data.de/Portals/0/NEPS/Datenzentrum/Forschungsdaten/SC4/ Methodenbericht_A46_A67_A83.pdf. Zugegriffen: 20. Jan. 2020.

Jerrim, J., \& Micklewright, J. (2014). Socio-economic gradients in children's cognitive skills: are crosscountry comparisons robust to who reports family background? European Sociological Review, 30, 766-781.

Kreuter, F., Eckman, S., Maaz, K., \& Watermann, R. (2010). Children's reports of parents' education level: Does it matter whom you ask and what you ask about? Survey Research Methods, 4, 127-138.

Kreuter, F., Maaz, K., \& Watermann, R. (2006). Der Zusammenhang zwischen der Qualität von Schülerangaben zur sozialen Herkunft und den Schulleistungen. In K.-S. Rehberg (Hrsg.), Soziale Ungleichheit, kulturelle Unterschiede: Verhandlungen des 32. Kongresses der Deutschen Gesellschaft für Soziologie in München (S. 3465-3478). Frankfurt a. M.: Campus.

Lien, N., Friestad, C., \& Klepp, K. I. (2001). Adolescents' proxy reports of parents' socioeconomic status: how valid are they? Journal of Epidemiology \& Community Health, 55, 731-737.

Lindemann, K., \& Gangl, M. (2019). Parental unemployment and the transition to vocational training in Germany: interaction of household and regional sources of disadvantage. European Sociological Review, 35(5), 684-700.

Maaz, K., Kreuter, F., \& Watermann, R. (2006). Schüler als Informanten? Die Qualität von Schülerangaben zum sozialen Hintergrund. In J. Baumert, P. Stanat \& R. Watermann (Hrsg.), Herkunftsbedingte Disparitäten im Bildungswesen: Differenzielle Bildungsprozesse und Probleme der Verteilungsgerechtigkeit (S. 31-59). Wiesbaden: Springer VS. 
Maniaci, M.R., \& Rogge, R.D. (2014). Caring about carelessness: participant inattention and its effects on research. Journal of Research in Personality, 48, 61-83.

Meade, A. W., \& Craig, S. B. (2012). Identifying careless responses in survey data. Psychological Methods, 17, 437-455.

Miyamoto, A., Seuring, J., \& Kristen, C. (2020). Immigrant students' achievements in light of their educational aspirations and academic motivation. Journal of Ethnic and Migration Studies, 46(7), $1348-1370$

Mize, T. D., Doan, L., \& Long, J.S. (2019). A general framework for comparing predictions and marginal effects across models. Sociological Methodology, 49(1), 152-189.

NEPS (2013). Startkohorte 4: Klasse 9 (SC4). Wellen 1 und 2 Erhebungsinstrumente (Feldversion). Bamberg: NEPS. https://www.neps-data.de/Portals/0/NEPS/Datenzentrum/Forschungsdaten/SC4/ Feldversionen/SC4_Q_w1-2_de.pdf. Zugegriffen: 20. Jan. 2020.

NEPS (2018). Informationen zur Kompetenztestung. NEPS Startkohorte 4 - Klasse 9. Schule und Ausbildung - Bildung von Schülerinnen und Schülern ab Klassen Stufe 9. 1. Welle: 9. Jahrgangsstufe. Bamberg: NEPS.

Nusser, L., Heydrich, J., Carstensen, C.H., Artelt, C., \& Weinert, S. (2016). Validity of survey data of students with special educational needs-results from the National Educational Panel Study. In H.P. Blossfeld, J. von Maurice, M. Bayer \& J. Skopek (Hrsg.), Methodological issues of longitudinal surveys (S. 251-266). Wiesbaden: Springer VS.

OECD (2017). PISA 2015 technical report. Paris: OECD Publishing.

Paternoster, R., Brame, R., Mazerolle, P., \& Piquero, A. (1998). Using the correct statistical test for the equality of regression coefficients. Criminology, 36, 859-866.

Rammstedt, B., \& John, O. P. (2005). Kurzversion des Big Five Inventory (BFI-K): Entwicklung und Validierung eines ökonomischen Inventars zur Erfassung der fünf Faktoren der Persönlichkeit. Diagnostica, 51, 195-206.

Ridolfo, H., \& Maitland, A. (2011). Factors that influence the accuracy of adolescent proxy reporting of parental characteristics: a research note. Journal of adolescence, 34, 95-103.

StataCorp (Hrsg.). (2017). Stata: release 15. Statistical software. College Station: StataCorp LLC.

Steinhauer, H. W., \& Zinn, S. (2016). NEPS technical report for weighting: weighting the sample of starting cohort 4 of the national educational panel study (wave 1 to 6) (NEPS Survey Paper No. 2). Bamberg: NEPS.

Tourangeau, R., Rips, L. J., \& Rasinski, K. (2000). The psychology of survey response. Cambridge: Cambridge University Press.

Vanette, D.L., \& Krosnick, J.A. (2014). Answering questions: a comparison of survey Satisficingand mindlessness. In A. Ie, C.T. Ngnoumen \& E. J. Langer (Hrsg.), The Wiley Blackwell handbook of mindfulness (Bd. 1-2, S. 312-327). Chichester: John Wiley \& Sons.

Vedel, A. (2014). The big five and tertiary academic performance: a systematic review and meta-analysis. Personality and Individual Differences, 71, 66-76.

West, P., Sweeting, H., \& Speed, E. (2001). We really do know what you do: a comparison of reports from 11 year olds and their parents in respect of parental economic activity and occupation. Sociology, 35, 539-559.

Wirtz, M.A., \& Caspar, F. (2002). Beurteilerübereinstimmung und Beurteilerreliabilität: Methoden zur Bestimmung und Verbesserung der Zuverlässigkeit von Einschätzungen mittels Kategoriensystemen und Ratingskalen. Göttingen: Hogrefe.

Wittrock, J., Kimmel, L., Hunscher, B., \& Le, K. T. (2017). Proxy reporting in education surveys: factors influencing accurate reporting in the 2012 Qatar Education Study. International Journal of Social Research Methodology, 20, 737-748. 